\title{
Bioindication of heavy metals using bryophyte communities in the Songtao manganese carbonate ore region, China
}

Chunmei He ( $18383205039 @ 163 . c o m)$

Guizhou Normal University

Zhaohui Zhang

Guizhou Normal University

Zhihui Wang

Guizhou Normal University

Kuangzheng Shi

Guizhou Normal University

Qimei Wu

Guizhou Normal University

Dengfu Wang

Guizhou Normal University

\section{Research Article}

Keywords: bryophyte communities, heavy metals, manganese carbonate, species diversity, indicator species

Posted Date: March 1st, 2021

DOI: https://doi.org/10.21203/rs.3.rs-202426/v1

License: (c) (i) This work is licensed under a Creative Commons Attribution 4.0 International License. Read Full License 


\section{Abstract}

Mining and mineral processing are often associated with heavy metal pollution. To reflect the environmental conditions of manganese carbonate ore deposits and of the area surrounding an electrolytic manganese plant, we analyzed the composition and species diversity of bryophyte communities and determined the differences in bryophyte communities related to the content of heavy metals in the substrate. We found that Pottiaceae and Bryaceae were the dominant families in the study area. The species abundance index of bryophyte communities at different locations was in order: vicinity of the mine $>$ mine site $>$ herb-shrubland > electrolytic plant > electrolytic waste dump. The species diversity of bryophyte communities at different locations was in order: herb-shrubland > vicinity of the mine > electrolytic waste dump $>$ mine site > electrolytic plant. Significant differences between heavy metal contents of the substrate were found among the different locations with the exception of $\mathrm{Co}(p<0.05)$. Mn contents were significantly different at each location $(p<$ 0.05). CCA showed that $\mathrm{Mn}, \mathrm{Cd}, \mathrm{Hg}$, and $\mathrm{Pb}$ content in the substrate were the major factors affecting the characteristics of the bryophyte communities. From the combination of CCA with the correlation heat map, it can be inferred that $\mathrm{Hg}, \mathrm{Mn}$, and $\mathrm{Cu}$ all have an adverse effect on bryophyte communities in the vicinity of the mine and in the herb-shrubland. Thus the determination of the characteristics of bryophyte communities allows ready identification of the impact of heavy metals on the environment, in turn providing a mechanism for decision-making in terms of pollution management and biodiversity protection.

\section{Introduction}

Manganese is one of the most important raw materials in the iron and steel industry where it is used as a deoxidizer and desulfurizer in production of iron, steel and industrial alloys (CMDEC, 1989). Manganese is principally sourced from manganese ore. China's iron and steel industry has developed rapidly in recent years driving an increasing demand for, and exploitation of, manganese ore that is increasing every year, and in the process, posing a serious threat to the natural environment and to the health of residents living in proximity to the manganese mines. China has vast deposits of manganese ore, but resources of low-grade ore are far more extensive than deposits of high-grade ore. More than $70 \%$ of the manganese ore deposits in China are comprised of manganese carbonate with less than $25 \%$ manganese (Liu et al., 2015; Deng et al., 2019), with the result that production of a given quantity of manganese requires the processing of significantly more manganese ore, eventuating in the production of even more slag and waste rock and further damage to the environment. When slag and waste rock are exposed to the environment, weathering and eluviation release heavy metals into the soil, water and atmosphere, ultimately ending in the food chain where they pose a serious threat to human health (Muhammad et al., 2011). The Songtao manganese mine is a very large mine with substantial processing capacity located in the core area of China's "manganese triangle", an area with the largest manganese carbonate ore reserves in China (Liu et al., 2019). In addition to the direct impact on the local environment, heavy metals can also follow watercourses to reach other areas, vastly expanding their impact range. In the study area, the Huayuan River flows through the electrolytic manganese plant, then into the Qiushui River, a tributary of Yuanjiang River, impacting a much greater area than the immediate vicinity of the mine.

When compared to vascular plants, bryophytes are extremely sensitive to environmental changes. The leaf structure of most bryophytes is relatively simple, composed of a single layer or, at most, few layers of cells, and in the absence of a waxy cuticle on the leaf surface, environmental pollutants can readily enter both upper and lower leaf surfaces where they remain (Wu, 1998; Wu et al., 2001). Bryophytes also have a capacity for robust cation exchange (Büscher et al., 1990) and can adsorb metal ions and metal particles from the surrounding environment into the leaves (Richardson, 1981). Thus, bryophytes are often used as indicator plants to monitor environmental pollutants; not only the degree of pollution, but the nature of, and changes in, pollutants. The sensitivity of bryophytes to their environment varies depending on their substrates. Epiphytic bryophytes are more sensitive to atmospheric conditions than either lithophytic or terrestrial 
bryophytes (Rao, 1982), both of which have the potential to entrap greater quantities of soil and dust particles than epiphytic bryophytes (Bargagli, 2016).

At present, there are two procedures used to determine environmental pollution using bryophytes: firstly, using moss bags (Wu et al., 2008; Salo et al., 2012; Vuković et al., 2013); secondly the collection and analysis of bryophytes growing in an area in question. Content of heavy metals determined in bryophytes of the same species, or the same genus, can be used to reflect the pollution status of a given area (Vincent et al., 2001; Gerdol et al., 2002; Schintu et al., 2005; Guo et al., 2017; Liu et al., 2018; Cowden et al., 2019) and characteristics of bryophyte communities can also be used to monitor environmental pollution (Denayer et al., 1999; Liu et al., 2011; Wang et al., 2015). In harsh environments such as mines, caves and areas of rocky desertification, bryophyte communities are often used to monitor heavy metal pollution (Liu et al., 2018) because of the difficulty of finding and identifying bryophytes of the same species or the same genus in a relatively small-scale area and the problem of locating sufficient materials for investigation. However, the species diversity of a bryophyte community can adequately reflect levels of environmental pollution (Yang et al., 2011). Changes in environmental conditions transform the structure and functional characteristics of animal and plant communities and heavy metals pollution stress reduces the stability of the community and also species diversity (Chen et al., 1989; Xu et al., 2004; Yang et al., 2011; Li et al., 2020). In Guizhou Province, bryophytes have been used to monitor heavy metal pollution in mercury, gold, copper and bauxite mines. The objectives of this study are: 1) to understand the composition, richness and species diversity of bryophyte communities in different locations associated with manganese carbonate mining and processing; 2) to determine the heavy metal content of the substrates underlying bryophyte communities in the vicinity of the mining operations; 3 ) to explore whether composition of bryophyte communities on heavily polluted mining processing areas can be used to determine the heavy metal composition of the underlying substrates.

\section{Materials And Methods}

\subsection{Study area}

The study area is located in Songtao Miaozu Autonomous County, Tongren City, Guizhou Province, China (Fig.1). The region has a mild-subtropical, humid, monsoonal climate with very high rainfall, high temperatures in summer, and very low temperatures in winter. The mean annual temperature is $16.5^{\circ} \mathrm{C}$ : the daily average temperature in July is $27.3^{\circ} \mathrm{C} ; 4.3^{\circ} \mathrm{C}$ in January. The mean annual precipitation is $1378.3 \mathrm{~mm}$, with a mean of 183 rainy days each year, and 1228 mean annual hours of sunshine duration. The mine is located on the passive continental margin of the southeastern boundary of the Yangtze Block. The manganese ore is mainly composed of rhodochrosite $\left(\mathrm{MnCO}_{3}\right)$ and calcimangite, followed by manganese dolomite, manganese-bearing calcite, pyrite, chalcopyrite, quartz, feldspar, clay minerals, carbonaceous organic matter and a small quantity of sulfate including gypsum, barite and other heavy minerals. The content of $\mathrm{MnO}_{2}$ ranges from $11.02 \% \sim 32.71 \%$; the content of $\mathrm{Fe}_{2} \mathrm{O}_{3}, \mathrm{P}_{2} \mathrm{O}_{5}$ and $\mathrm{SiO}_{2}$ are $2.04 \% \sim 7.48 \%, 0.08 \% \sim 0.43 \%$ and $20.90 \% \sim$ $65.90 \%$ respectively (Gao et al., 2018; He et al., 2013).

Table 1. Basic information for the five sampling locations

\begin{tabular}{|lllll|}
\hline Location & Longitude & Latitude & Elevation & Sampling plot \\
\hline Vicinity of the manganese mine (a) & $109^{\circ} 06^{\prime} 42^{\prime \prime} \mathrm{E}$ & $28^{\circ} 06^{\prime} 42^{\prime \prime} \mathrm{N}$ & $525.20 \pm 3 \mathrm{~m}$ & plots a1-a5 \\
\hline Manganese mine site (b) & $109^{\circ} 06^{\prime} 42^{\prime \prime} \mathrm{E}$ & $28^{\circ} 06^{\prime} 42^{\prime \prime} \mathrm{N}$ & $525.20 \pm 3 \mathrm{~m}$ & plots b1-b5 \\
\hline Electrolytic plant (c) & $109^{\circ} 11^{\prime} 57^{\prime \prime} \mathrm{E}$ & $28^{\circ} 11^{\prime} 01^{\prime \prime} \mathrm{N}$ & $374.88 \pm 6 \mathrm{~m}$ & plots c1-c5 \\
\hline Electrolytic waste dump (d) & $109^{\circ} 10^{\prime} 39^{\prime \prime} \mathrm{E}$ & $28^{\circ} 11^{\prime} 19^{\prime \prime} \mathrm{N}$ & $401.95 \pm 4 \mathrm{~m}$ & plots d1-d5 \\
\hline Herb-shrubland (e) & $109^{\circ} 10^{\prime} 35^{\prime \prime} \mathrm{E}$ & $28^{\circ} 14^{\prime} 30^{\prime \prime} \mathrm{N}$ & $514.81 \pm 6 \mathrm{~m}$ & plots e1-e5 \\
\hline
\end{tabular}


2.2 Sample collection and processing Samples of substrate soil from $1 \mathrm{~cm} 3 \mathrm{~cm}$ deep were collected from each plot and packed into polyethylene plastic bags. A substrate sample consisted of a mixture of 5 soil samples collected from each plot, providing a total of 25 substrate samples. Plant roots and gravel in the samples were removed after air drying in the shade, the remainder sent to the laboratory, grind and homogenized, and stored in sealed bags for determination of heavy metals. 2.3 Determination of heavy metalsSubstrate samples were digested by an automatic digester (AutoDigiBlock, Labtech, Beijing). $0.2 \mathrm{~g}$ of each substrate sample was weighed, put into a PTFE digestion tube which in turn was placed in a graphite digestion hole, subsequently, a test was conducted with a blank. After the samples in the PTFE digestion tube were moistened with water, $6 \mathrm{ml}$ nitric acid (68.0\% 70.0\%, UPS), $3 \mathrm{ml}$ hydrofluoric acid (48.8\% 49.2\%, UPS) and $1 \mathrm{ml}$ perchloric acid $(70.0 \% \sim 72.0 \%$, GR) were added to the digestion tube, which was then well shaken and the tube cover tightened. The digester was first programmed to $100^{\circ} \mathrm{C}$ to heat the digestion solution and reflux for $1 \mathrm{~h}$, then cooled to room temperature and the tube cover opened. It was then programmed to $140{ }^{\circ} \mathrm{C}$ to force out the acid until $0.5 \sim 1 \mathrm{ml}$ of the digestion solution remained. Finally, the inner wall of the digestion tube was cleaned with a small amount of ultrapure water and diluted with $2 \%$ nitric acid to return the volume to $50 \mathrm{ml}$. The Inductively Coupled Plasma Optical Emission Mass Spectrometer (NexION 300X, PerkinElmer, USA) was used for the determination of Mn, Fe, Cr, Co, Ni, Cu, Zn, Cd, Hg, $\mathrm{Pb}$ in the digestion solution.In the determination process, soil standard GBW07405 (GSS-11) was analyzed to check the accuracy and precision of each metal analysis.

\subsection{Data analysis}

The Abundance index (Zuo, 1990), Shannon-Wiener diversity index, Simpson diversity index, and Pielou evenness index were used to determine the characteristics of the bryophyte communities at each location. This was calculated by the cover to represent the number of individual bryophyte species. The calculation formulas are as follows: Please see

\section{formulas 1 - 4 in the supplementary files.}

$m$ is the number of species in a region; $P_{i}$ is the important value of species, $P_{i}=N_{i} / N, N_{i}$ is the number of the $i$ species among $m$ species, and $N$ is the total number of $m$ species.

ArcGIS was used to draw the sampling area map; the difference analysis of data was completed in Spss 21.0; heavy metal and bryophyte species data were assessed using by Microsoft Excel 2016; diversity data were calculated by 'vegan' package of R (Version 4.0.1); the indicator species was determined by the R package 'labdsv', statistical analysis was performed using the 'ggplot' package, 'corrplot' package and 'pheatmap' package in R (Version 4.0.1) software, CCA analysis was performed using Canoco5.0 software.

\section{Results}

\subsection{Characteristics of the bryophyte communities 3.1.1 Composition of bryophyte communities}

A total of 110 bryophyte species, 54 genera in 25 families were recorded at the five locations. These included six liverwort species from six genera in five families and 105 moss species from 48 genera in 20 families. The dominant families were Pottiaceae, Bryaceae and Hypnaceae, accounting for $28.8 \%, 16.2 \%$ and $12.6 \%$ of species, respectively. There was a considerable difference in the number of species, genera and families at each location (Fig. 2A): most occurred in the vicinity of the manganese mine, (38 species, 32 genera, 17 families); fewest were recorded at the electrolytic waste dump (14 genera, 4 families); and the lowest number of species at the electrolytic plant. The Abundance index for the five locations was similar to the distribution of genera and families, with the highest Abundance index recorded in the vicinity 
of the mine and the lowest at the electrolytic waste dump. The species composition of bryophytes at the family level was similar in the vicinity of the mine and at the mine site area. Hypnaceae, Pottiaceae and Leucobryaceae accounted for the largest proportion of species numbers in the vicinity of the mine with percentages of $38.67 \%, 17.51 \%$ and $9.06 \%$, respectively (Fig. 2B). At the mine site, Polytrichaceae, Ditrichaceae and Pottiaceae accounted for $35.65 \%, 16.95 \%$ and $12.92 \%$, respectively. The electrolytic plant and the electrolytic waste dump were characterized mainly by Pottiaceae and

Bryaceae, which accounted for $90.5 \%$ and $98.9 \%$, respectively. Hypnaceae species were widely distributed in the vicinity of the mine, at the mine site and in the herb-shrubland area, but was not found near either the electrolytic plant or the electrolytic waste dump. Short turf life-forms accounted for $55.82 \%$ of bryophyte taxa at all five locations, comprising $39.47 \%, 67.65 \%, 83.33 \%, 92.86 \%$ and $35.14 \%$ in the vicinity of the mine, at the mine site itself, at the electrolytic plant, the electrolytic waste dump and in the herb-shrubland, respectively.

Five bryophyte species were determined as indicator species for the five locations. Campylopus coreensis, Pogonatum neesii, Barbula ehrenbergii, Trichostomum brachydontium and Pseudosymblepharis angustata were the indictors for the vicinity of the mine, mine site, electrolytic plant, electrolytic waste dump and herb-shrubland, respectively (Table A1).

\subsubsection{Species diversity of bryophyte communities in the five locations}

Three alpha diversity indices, Shannon-Wiener diversity index, Simpson diversity index, and Pielou evenness index, were calculated to evaluate the diversity of the bryophyte communities (Fig. 2C). The alpha diversity values in herb-shrubland were higher than those of the other four locations with small variation in range and relatively uniform distribution. The alpha diversity values of the electrolytic plant were the lowest, with a large variation range and uneven distribution. In general, the alpha diversity values of each location were observed as herb-shrubland $>$ vicinity of the mine $>$ electrolytic waste dump > mine site > electrolytic plant.

\subsection{Analysis of heavy metal contents in substrates underlying bryophyte communities}

The total concentrations of ten heavy metals in the substrates underlying the bryophyte communities were measured. Heavy metal contents for each location are the mean of the five plots shown in Table 2. There were significant differences in $\mathrm{Mn}$ content among the five locations $(\mathrm{P}<0.05)$, in the order: electrolytic plant $>$ mine site > vicinity of the mine > electrolytic waste dump > herb-shrubland. Except herb-shrubland, Mn contents in the vicinity of the mine, at the mine site, at the electrolytic plant, and at the electrolytic waste dump, were 4.80, 7.02, 9.79 and 2.28 times higher than the background values for soil in Guizhou Province, respectively. The contents of all ten heavy metals at the electrolytic plant were higher than the Guizhou background values. With the exception of Co and $\mathrm{Hg}$, the contents of other heavy metals at the electrolytic plant were higher than at any other of the five locations. The contents of $\mathrm{Ni}, \mathrm{Cu}, \mathrm{Zn}$ and $\mathrm{Pb}$ at the electrolytic plant were significantly different from the other four locations $(P<0.05)$, but there were no significant differences in $\mathrm{Ni}, \mathrm{Zn}$ and $\mathrm{Pb}$ contents in the vicinity of the mine, at the mine, at the electrolytic waste dump and in the herbshrubland $(P>0.05)$. With the exception of $\mathrm{Cr}$, the difference in metals contents between the vicinity of the mine and the mine site itself, was not significant, and with the exception of $\mathrm{Cd}$, the contents of other heavy metals at the two locations were higher than Guizhou background values.

Correlation analysis showed that there were significant positive correlations among $\mathrm{Co}, \mathrm{Mn}, \mathrm{Fe}, \mathrm{Zn}, \mathrm{Cr}, \mathrm{Cu}, \mathrm{Ni}$ and $\mathrm{Pb}(\mathrm{P}<$ 0.01 or $P<0.05$ ) (Fig. 3), while the correlations between $\mathrm{Hg}$ and other heavy metals were not significant $(\mathrm{P}>0.05)$. Cd element had significant positive correlation with $\mathrm{Pb}$ and $\mathrm{Cu}(\mathrm{P}<0.01$ or $\mathrm{P}<0.05)$, but had no significant correlation with other heavy metal elements $(P>0.05)$. 
Table 2

Contents of heavy metals in the substrate underlying bryophyte communities

\begin{tabular}{|c|c|c|c|c|c|c|}
\hline Element & $\begin{array}{l}\text { Vicinity of the } \\
\text { mine }\end{array}$ & Mine site & $\begin{array}{l}\text { Electrolytic } \\
\text { plant }\end{array}$ & $\begin{array}{l}\text { Electrolytic waste } \\
\text { dump }\end{array}$ & $\begin{array}{l}\text { Herb- } \\
\text { shrubland }\end{array}$ & $\begin{array}{l}\text { Guizhou background } \\
\text { values }\end{array}$ \\
\hline $\begin{array}{l}\mathrm{Mn}\left(\mathrm{g} \cdot \mathrm{kg}^{-}\right. \\
\left.{ }^{1}\right)\end{array}$ & $3.81 \pm 0.48 b$ & $\begin{array}{l}5.57 \pm \\
0.56 \mathrm{ab}\end{array}$ & $\begin{array}{l}7.77 \pm \\
0.69 a\end{array}$ & $1.81 \pm 0.10 c$ & $\begin{array}{l}0.75 \pm \\
0.05 \mathrm{~d}\end{array}$ & 0.794 \\
\hline $\begin{array}{l}\mathrm{Fe}\left(\mathrm{g} \cdot \mathrm{kg}^{-}\right. \\
\left.{ }^{1}\right)\end{array}$ & $52.89 \pm 2.98 b$ & $\begin{array}{l}55.81 \pm \\
0.37 a b\end{array}$ & $\begin{array}{l}68.94 \pm \\
13.28 \mathrm{a}\end{array}$ & $41.72 \pm 11.30 \mathrm{~b}$ & $\begin{array}{l}43.40 \pm \\
4.17 \mathrm{~b}\end{array}$ & 4.17 \\
\hline $\begin{array}{l}\mathrm{Cr}(\mathrm{mg} \cdot \mathrm{kg} \\
-1)\end{array}$ & $\begin{array}{l}97.43 \pm \\
6.81 \mathrm{bc}\end{array}$ & $\begin{array}{l}108.50 \pm \\
5.38 a b\end{array}$ & $\begin{array}{l}126.01 \pm \\
8.57 a\end{array}$ & $69.82 \pm 6.05 c$ & $\begin{array}{l}79.47 \pm \\
6.77 \mathrm{bc}\end{array}$ & 95.9 \\
\hline $\begin{array}{l}\text { Co (mg.kg } \\
-1)\end{array}$ & $\begin{array}{l}38.52 \pm \\
11.55 a\end{array}$ & $\begin{array}{l}37.53 \pm \\
4.58 a\end{array}$ & $\begin{array}{l}37.90 \pm \\
4.59 a\end{array}$ & $19.84 \pm 2.63 a$ & $\begin{array}{l}18.45 \pm \\
2.03 a\end{array}$ & 19.2 \\
\hline $\begin{array}{l}\mathrm{Ni}(\mathrm{mg} \cdot \mathrm{kg} \\
-1)\end{array}$ & $52.67 \pm 4.74 b$ & $\begin{array}{l}40.51 \pm \\
3.23 b\end{array}$ & $\begin{array}{l}80.53 \pm \\
4.29 a\end{array}$ & $51.47 \pm 8.45 b$ & $\begin{array}{l}43.51 \pm \\
4.87 \mathrm{~b}\end{array}$ & 39.1 \\
\hline $\begin{array}{l}\text { Cu (mg.kg } \\
-1)\end{array}$ & $46.18 \pm 3.49 b$ & $\begin{array}{l}38.55 \pm \\
4.00 \mathrm{~b}\end{array}$ & $\begin{array}{l}76.41 \pm \\
6.17 a\end{array}$ & $34.74 \pm 6.18 b c$ & $\begin{array}{l}22.51 \pm \\
2.25 c\end{array}$ & 32.0 \\
\hline $\begin{array}{l}\mathrm{Zn}(\mathrm{mg} \cdot \mathrm{kg} \\
-1)\end{array}$ & $\begin{array}{l}160.72 \pm \\
15.57 b\end{array}$ & $\begin{array}{l}176.41 \pm \\
16.48 b\end{array}$ & $\begin{array}{l}257.38 \pm \\
29.10 a\end{array}$ & $151.95 \pm 37.69 b$ & $\begin{array}{l}148.70 \pm \\
18.57 b\end{array}$ & 99.5 \\
\hline $\begin{array}{l}\text { Cd (mg } \cdot k g \\
-1)\end{array}$ & $0.54 \pm 0.04 a b$ & $\begin{array}{l}0.36 \pm \\
0.04 \mathrm{~b}\end{array}$ & $\begin{array}{l}0.70 \pm \\
0.06 a\end{array}$ & $0.48 \pm 0.06 b$ & $\begin{array}{l}0.58 \pm \\
0.07 \mathrm{~b}\end{array}$ & 0.659 \\
\hline $\begin{array}{l}\mathrm{Hg}(\mathrm{mg} \cdot \mathrm{kg} \\
-1)\end{array}$ & $0.13 \pm 0.01 b$ & $\begin{array}{l}0.94 \pm \\
0.11 \mathrm{a}\end{array}$ & $\begin{array}{l}0.50 \pm \\
0.06 a\end{array}$ & $0.72 \pm 0.11 a$ & $\begin{array}{l}0.18 \pm \\
0.07 \mathrm{~b}\end{array}$ & 0.110 \\
\hline $\begin{array}{l}\mathrm{Pb}(\mathrm{mg} \cdot \mathrm{kg} \\
-1)\end{array}$ & $40.71 \pm 5.64 b$ & $\begin{array}{l}33.88 \pm \\
1.87 b\end{array}$ & $\begin{array}{l}146.02 \pm \\
18.57 a\end{array}$ & $53.59 \pm 6.47 b$ & $\begin{array}{l}38.48 \pm \\
3.15 b\end{array}$ & 35.2 \\
\hline
\end{tabular}

**indicates significant correlation at 0.01 level (bilateral); * indicates significant correlation at 0.05 level (bilateral).

\subsection{Relationship between bryophyte communities and heavy metal contents in substrate}

\subsubsection{CCA analysis of bryophyte species and heavy metal elements}

The 22 bryophyte species (total coverage > 1.0) selected for CCA analysis included five indicator species (Table A1). The significance of the contents of each heavy metal element in substrate and bryophyte species distribution was tested by envfit function in R software (999 simulation times were selected) and the heavy metal elements $\mathrm{Mn}, \mathrm{Cd}, \mathrm{Hg}, \mathrm{Pb}$ with significant explanatory amount were screened out (Table 3) and tested by Monte Carlo test with the significance less than 0.05. This indicated that the sorting results could accept the interpretation of the selected heavy metal elements on species distribution. The sequencing results are shown in Fig. 4.

CCA results for heavy metals indicated that the first principal component axis and the second principal component axis explained $82.15 \%$ and $65.91 \%$ of the bryophyte species composition, respectively. The total explanatory amount of $\mathrm{Mn}, \mathrm{Cd}$, $\mathrm{Hg}$, and $\mathrm{Pb}$ element was $23.9 \%$, accounting for $5.3 \%, 5.2 \%, 6.8 \%$ and $7.6 \%$, respectively. The distribution of bryophyte species on the ordination axis basically reflected the trend in variation of characteristics of plant spatial distribution with heavy metal contents. Most bryophytes were distributed in the negative direction of the second axis, while the species 
resistant to heavy metals were mainly distributed in the positive direction of the second axis. Didymodon tectorus (Did.tec), Barbula ehrenbergii (Bar.ehr), and Barbula propagulifera (Bar.pro) were distributed in regions with a high concentration of $\mathrm{Pb}$ and $\mathrm{Mn}$ in the substrate. Species distributed in the regions with high concentration of $\mathrm{Pb}$ and $\mathrm{Cd}$ included Hydrogonium majusculum (Hyd.maj), Didymodon constrictus (Dym.con), and Anomobryum julaceum (Ano.jul). The distribution of Bryum pallescens and Ditrichum pallidum were mainly affected by the concentration of $\mathrm{Hg}$ in the substrate. The distribution of bryophytes at the electrolytic plant was consistent with the variation direction of heavy metal elements, and the bryophytes at the electrolytic plant have a strong tolerance for heavy metals and were mainly affected by $\mathrm{Pb}$ and $\mathrm{Mn}$. Bryophyte distribution in the vicinity of the mine and in the herb-shrubland were opposite to the direction of heavy metals, indicating that the distribution of bryophyte at the two locations was negatively correlated with the heavy metal contents in the substrate and that the bryophytes at the two locations showed weak tolerance to heavy metals.

Table 3

Results of significance test of heavy metals

\begin{tabular}{|lllll|}
\hline Elements & CCA1 & CCA2 & $\mathbf{r}^{2}$ & $\operatorname{Pr}(>\mathrm{r})$ \\
\hline $\mathrm{Mn}$ & -0.10085 & 0.99490 & 0.5126 & 0.001998 ** \\
$\mathrm{Cd}$ & 0.23639 & 0.97166 & 0.3021 & 0.027972 * \\
\hline $\mathrm{Hg}$ & -0.67693 & 0.73604 & 0.3731 & 0.009990 ** \\
\hline $\mathrm{Pb}$ & 0.56690 & 0.82379 & 0.6139 & 0.000999 *** \\
\hline
\end{tabular}


Table A1

Occurrence, total coverage (TC), indicator values (IV) of bryophyte species in five locations. Species with indicator value $>$

0.5 are considered as the best indicators. Species with both high indicator value and a signifificant $p$ value $(p<0.05)$ are shown in bold.

\begin{tabular}{|c|c|c|c|c|c|c|c|c|c|c|c|}
\hline \multirow[t]{2}{*}{ No. } & \multirow[t]{2}{*}{ Family name } & \multirow[t]{2}{*}{ Species name } & \multirow[t]{2}{*}{ Abbreviation } & \multicolumn{5}{|c|}{ Location } & \multirow[t]{2}{*}{$\mathrm{TC}$} & \multirow[t]{2}{*}{ IV } & \multirow[t]{2}{*}{$p$} \\
\hline & & & & a & b & c & d & e & & & \\
\hline 1 & $\begin{array}{l}\text { Allisoniaceae } \\
\text { (R.M.Schust.ex } \\
\text { Grolle)Schljakov }\end{array}$ & $\begin{array}{l}\text { Calycularia crispula } \\
\text { Mitt*. }\end{array}$ & Cal. cri & 0 & 0 & 0 & 0 & + & 0.096 & - & - \\
\hline \multirow[t]{3}{*}{2} & $\begin{array}{l}\text { Bartramiaceae } \\
\text { Schwägr }\end{array}$ & $\begin{array}{l}\text { Philonotis hastata } \\
\text { (Duby) Wijk \& Marg. }\end{array}$ & Phi.has & 0 & + & 0 & 0 & 0 & 0.07 & - & - \\
\hline & & $\begin{array}{l}\text { Philonotis mollis } \\
\text { (Dozy \& Molk.) Mitt. }\end{array}$ & Phi.mol & + & + & 0 & + & 0 & 0.9099 & - & - \\
\hline & & $\begin{array}{l}\text { Philonotis turneriana } \\
\text { (Schwägr.) Mitt. }\end{array}$ & Phi.tur & 0 & + & + & 0 & 0 & 0.7648 & - & - \\
\hline \multirow[t]{6}{*}{3} & $\begin{array}{l}\text { Brachytheciaceae } \\
\text { Schimp. }\end{array}$ & $\begin{array}{l}\text { Brachythecium } \\
\text { perminusculum } \\
\text { Müll. Hal. }\end{array}$ & Bra.per & 0 & 0 & 0 & 0 & + & 0.1001 & - & - \\
\hline & & $\begin{array}{l}\text { Brachythecium } \\
\text { reflexum (Stark.) } \\
\text { Schimp. }\end{array}$ & Bra.ref & + & 0 & 0 & 0 & + & 0.256 & - & - \\
\hline & & $\begin{array}{l}\text { Brachythecium } \\
\text { rutabulum (Hedw.) } \\
\text { Bruch \& Schimp. }\end{array}$ & Bra.rut & + & 0 & 0 & 0 & 0 & 0.172 & - & - \\
\hline & & $\begin{array}{l}\text { Eurhynchium laxirete } \\
\text { Broth. }\end{array}$ & Eur.lax & + & 0 & 0 & 0 & 0 & 0.434 & - & - \\
\hline & & $\begin{array}{l}\text { Rhynchostegium } \\
\text { fauriei Cardot }\end{array}$ & Rhy.fau & 0 & 0 & + & 0 & 0 & 0.084 & - & - \\
\hline & & $\begin{array}{l}\text { Rhynchostegium } \\
\text { inclinatum (Mitt.) } \\
\text { A.Jeag. }\end{array}$ & Rhy.inc & + & 0 & 0 & 0 & 0 & 0.14 & - & - \\
\hline \multirow[t]{2}{*}{4} & $\begin{array}{l}\text { Bruchiaceae } \\
\text { Schimp. }\end{array}$ & $\begin{array}{l}\text { Trematodon } \\
\text { ambiguus(Hedw.) } \\
\text { Hornsch. }\end{array}$ & Tre.amb & 0 & + & 0 & 0 & 0 & 0.1518 & - & - \\
\hline & & $\begin{array}{l}\text { Trematodon } \\
\text { longicollis Michx. }\end{array}$ & Tre.lon & 0 & + & 0 & 0 & 0 & 0.128 & - & - \\
\hline \multirow[t]{4}{*}{5} & Bryaceae Schwägr. & $\begin{array}{l}\text { Anomobryum } \\
\text { gemmigerum Broth. }\end{array}$ & Ano.gem & 0 & 0 & 0 & 0 & + & 0.063 & - & - \\
\hline & & $\begin{array}{l}\text { Anomobryum } \\
\text { julaceum (Gärtn., } \\
\text { Meyer \& Scherb.) } \\
\text { Schimp. }\end{array}$ & Ano.jul & 0 & 0 & + & + & 0 & 3.0877 & - & - \\
\hline & & $\begin{array}{l}\text { Brachymenium exile } \\
\text { (Dozy \&. Molk.) } \\
\text { Bosch \& Sande Lac. }\end{array}$ & Bra.exi & 0 & 0 & + & + & 0 & 0.5265 & - & - \\
\hline & & $\begin{array}{l}\text { Brachymenium } \\
\text { leptophyllum (Müll. } \\
\text { Hal.) A. Jaeger. }\end{array}$ & Bra.lep & 0 & 0 & 0 & + & 0 & 0.354 & - & - \\
\hline
\end{tabular}

Note: “»” represent liverworts 


\begin{tabular}{|c|c|c|c|c|c|c|c|c|c|c|c|}
\hline \multirow[t]{2}{*}{ No. } & \multirow[t]{2}{*}{ Family name } & \multirow[t]{2}{*}{ Species name } & \multirow[t]{2}{*}{ Abbreviation } & \multicolumn{5}{|c|}{ Location } & \multirow[t]{2}{*}{ TC } & \multirow[t]{2}{*}{ IV } & \multirow[t]{2}{*}{$p$} \\
\hline & & & & a & b & c & d & e & & & \\
\hline & & $\begin{array}{l}\text { Bryum argenteum } \\
\text { Hedw. }\end{array}$ & Bry.arg & + & 0 & + & + & 0 & 0.6567 & - & - \\
\hline & & $\begin{array}{l}\text { Bryum atrovirens } \\
\text { Brid. }\end{array}$ & Bry.atr & 0 & 0 & 0 & + & 0 & 0.2747 & - & - \\
\hline & & $\begin{array}{l}\text { Bryum billarderi } \\
\text { Schwägr. }\end{array}$ & Bry.bil & 0 & 0 & 0 & + & + & 0.279 & - & - \\
\hline & & $\begin{array}{l}\text { Bryum blindii Bruch } \\
\text { \& Schimp. }\end{array}$ & Bry.bli & 0 & 0 & + & 0 & 0 & 0.0512 & - & - \\
\hline & & $\begin{array}{l}\text { Bryum } \\
\text { bornholmense } \\
\text { Winkelm. \& Ruthe. }\end{array}$ & Bry.bor & 0 & 0 & + & 0 & 0 & 0.1961 & - & - \\
\hline & & $\begin{array}{l}\text { Bryum capillare } \\
\text { Hedw. }\end{array}$ & Bry.cap & + & 0 & 0 & 0 & 0 & 0.136 & - & - \\
\hline & & $\begin{array}{l}\text { Bryum coronatum } \\
\text { Schwägr. }\end{array}$ & Bry.cor & 0 & 0 & + & 0 & 0 & 0.3251 & - & - \\
\hline & & $\begin{array}{l}\text { Bryum dichotomum } \\
\text { Hedw. }\end{array}$ & Bry.dic & 0 & 0 & + & + & 0 & 0.7386 & - & - \\
\hline & & $\begin{array}{l}\text { Bryum leptocaulon } \\
\text { Cardot. }\end{array}$ & Bry.lep & 0 & 0 & 0 & + & 0 & 0.116 & - & - \\
\hline & & $\begin{array}{l}\text { Bryum pallescens } \\
\text { Schleicher ex } \\
\text { Schwägr. }\end{array}$ & Bry.pal & 0 & 0 & + & + & + & 1.5712 & - & - \\
\hline & & $\begin{array}{l}\text { Bryum paradoxum } \\
\text { Schwägr. }\end{array}$ & Bry.par & 0 & 0 & 0 & + & 0 & 0.1931 & - & - \\
\hline & & $\begin{array}{l}\text { Bryum } \\
\text { pseudotriquetrum } \\
\text { (Hedw.) Gaertn. }\end{array}$ & Bry.pse & 0 & 0 & + & 0 & 0 & 0.205 & - & - \\
\hline & & $\begin{array}{l}\text { Bryum thomsonii } \\
\text { Mitt. }\end{array}$ & Bry.tho & 0 & + & 0 & + & 0 & 0.108 & - & - \\
\hline & & $\begin{array}{l}\text { Bryum tuberosum } \\
\text { Mohamed \& } \\
\text { Damanhuri }\end{array}$ & Bry.tub & 0 & + & 0 & 0 & 0 & 0.018 & - & - \\
\hline & & $\begin{array}{l}\text { Philonotis falcata } \\
\text { (Hook) Mitt. }\end{array}$ & Phi.fal & + & 0 & 0 & 0 & 0 & 0.032 & - & - \\
\hline 6 & $\begin{array}{l}\text { Dicranaceae } \\
\text { Schimp. }\end{array}$ & $\begin{array}{l}\text { Microdus sinensis } \\
\text { Herz }\end{array}$ & Mic.sin & + & 0 & 0 & 0 & 0 & 0.002 & - & - \\
\hline 7 & $\begin{array}{l}\text { Dicranellaceae } \\
\text { M.Stech }\end{array}$ & $\begin{array}{l}\text { Dicranella } \\
\text { heteromalla (Hedw.) } \\
\text { Schimp. }\end{array}$ & Dic.het & 0 & + & 0 & 0 & 0 & 0.06 & - & - \\
\hline & $\begin{array}{l}\text { Dicranellaceae } \\
\text { M.Stech }\end{array}$ & $\begin{array}{l}\text { Dicranella subulata } \\
\text { (Hedw.) Schimp. }\end{array}$ & Dic.sub & + & + & + & 0 & + & 1.2176 & - & - \\
\hline & $\begin{array}{l}\text { Dicranellaceae } \\
\text { M.Stech }\end{array}$ & $\begin{array}{l}\text { Dicranella varia } \\
\text { (Hedw.) Schimp }\end{array}$ & Dic.var & 0 & 0 & 0 & + & 0 & 0.0969 & - & - \\
\hline
\end{tabular}

Note: “»” represent liverworts 


\begin{tabular}{|c|c|c|c|c|c|c|c|c|c|c|c|}
\hline \multirow[t]{2}{*}{ No. } & \multirow[t]{2}{*}{ Family name } & \multirow[t]{2}{*}{ Species name } & \multirow[t]{2}{*}{ Abbreviation } & \multicolumn{5}{|c|}{ Location } & \multirow[t]{2}{*}{$\mathrm{TC}$} & \multirow[t]{2}{*}{ IV } & \multirow[t]{2}{*}{$p$} \\
\hline & & & & $\mathbf{a}$ & b & c & d & e & & & \\
\hline \multirow[t]{2}{*}{8} & \multirow[t]{2}{*}{ Ditrichaceae Limpr. } & $\begin{array}{l}\text { Ditrichum pallidum } \\
\text { (Hedw.) Hampe }\end{array}$ & Dit.pal & + & + & 0 & 0 & 0 & 2.8652 & - & - \\
\hline & & $\begin{array}{l}\text { Pleuridium } \\
\text { subulatum } \\
\text { (Hedw.)Rabenh. }\end{array}$ & Ple.sub & 0 & + & 0 & 0 & 0 & 0.108 & - & - \\
\hline \multirow[t]{5}{*}{9} & \multirow[t]{5}{*}{$\begin{array}{l}\text { Fissidentaceae } \\
\text { Schimp. }\end{array}$} & $\begin{array}{l}\text { Fissidens anomalus } \\
\text { Mont. }\end{array}$ & Fis.ano & 0 & 0 & 0 & 0 & + & 0.246 & - & - \\
\hline & & $\begin{array}{l}\text { Fissidens dubius } \mathrm{P} . \\
\text { Beauv. }\end{array}$ & Fis.dub & 0 & 0 & 0 & 0 & + & 0.7389 & - & - \\
\hline & & $\begin{array}{l}\text { Fissidens incognitus } \\
\text { Gangulee. }\end{array}$ & Fis.inc & 0 & 0 & + & 0 & 0 & 0.08 & - & - \\
\hline & & $\begin{array}{l}\text { Fissidens involutus } \\
\text { Wilson ex Mitt. }\end{array}$ & Fis.inv & 0 & 0 & + & 0 & 0 & 0.1122 & - & - \\
\hline & & $\begin{array}{l}\text { Fissidens taxifolius } \\
\text { Hedw. }\end{array}$ & Fis.tax & + & 0 & 0 & 0 & + & 0.039 & - & - \\
\hline 10 & $\begin{array}{l}\text { Funariaceae } \\
\text { Schwägr. }\end{array}$ & $\begin{array}{l}\text { Physcomitrium } \\
\text { sphaericum (Ludw.) } \\
\text { Fürnr. }\end{array}$ & Phy.sph & + & 0 & + & 0 & 0 & 0.1246 & - & - \\
\hline \multirow[t]{11}{*}{11} & \multirow[t]{11}{*}{$\begin{array}{l}\text { Hypnaceae } \\
\text { Schimp. }\end{array}$} & $\begin{array}{l}\text { Ctenidium hastile } \\
\text { (Mitt.) Lindb. }\end{array}$ & Cte.has & 0 & + & 0 & 0 & 0 & 0.16 & - & - \\
\hline & & $\begin{array}{l}\text { Gollania arisanensis } \\
\text { Sakurai }\end{array}$ & Gol.ari & 0 & 0 & 0 & 0 & + & 0.0375 & - & - \\
\hline & & $\begin{array}{l}\text { Gollania revoluta } \\
\text { Higuchi }\end{array}$ & Gol.rev & 0 & 0 & 0 & 0 & + & 0.045 & - & - \\
\hline & & $\begin{array}{l}\text { Gollania ruginosa } \\
\text { (Mitt.) Broth. }\end{array}$ & Gol.rug & 0 & 0 & 0 & 0 & + & 0.0693 & - & - \\
\hline & & $\begin{array}{l}\text { Hypnum calcicola } \\
\text { Ando }\end{array}$ & Hyp.cal & + & 0 & 0 & 0 & + & 3.8315 & - & - \\
\hline & & $\begin{array}{l}\text { Hypnum } \\
\text { leptothallum } \\
\text { (C.Muell.) Paris. }\end{array}$ & Hyp.lep & 0 & 0 & 0 & 0 & + & 1.3028 & - & - \\
\hline & & $\begin{array}{l}\text { Hypnum } \\
\text { oldhamii(Mitt.) A. } \\
\text { Jaeger. }\end{array}$ & Hyp.old & + & 0 & 0 & 0 & + & 2.8876 & - & - \\
\hline & & $\begin{array}{l}\text { Hypnum pallescens } \\
\text { (Hedw.) P.Beauv. }\end{array}$ & Hyp.pal & + & 0 & 0 & 0 & + & 0.9822 & - & - \\
\hline & & $\begin{array}{l}\text { Hypnum revolutum } \\
\text { (Mitt.) Lindb. }\end{array}$ & Hyp.rev & 0 & + & 0 & 0 & + & 1.2044 & - & - \\
\hline & & $\begin{array}{l}\text { Hypnum vaucheri } \\
\text { Lesq. }\end{array}$ & Hyp.vau & 0 & 0 & 0 & 0 & + & 0.184 & - & - \\
\hline & & $\begin{array}{l}\text { Pseudotaxiphyllum } \\
\text { densum (Cardot) Z. } \\
\text { Iwats. }\end{array}$ & Pse.den & + & + & 0 & 0 & 0 & 0.112 & - & - \\
\hline
\end{tabular}

Note: “*” represent liverworts 


\begin{tabular}{|c|c|c|c|c|c|c|c|c|c|c|c|}
\hline \multirow[t]{2}{*}{ No. } & \multirow[t]{2}{*}{ Family name } & \multirow[t]{2}{*}{ Species name } & \multirow[t]{2}{*}{ Abbreviation } & \multicolumn{5}{|c|}{ Location } & \multirow[t]{2}{*}{$\mathrm{TC}$} & \multirow[t]{2}{*}{ IV } & \multirow[t]{2}{*}{$p$} \\
\hline & & & & a & b & c & d & e & & & \\
\hline & & $\begin{array}{l}\text { Pseudotaxiphyllum } \\
\text { pohliaecarpum (Sull. } \\
\text { \& Lesq.) Z. Iwats. }\end{array}$ & Pse.poh & 0 & + & 0 & 0 & 0 & 0.317 & - & - \\
\hline & & $\begin{array}{l}\text { Taxiphyllum } \\
\text { aomoriense (Besch.) } \\
\text { Z. Iwats. }\end{array}$ & Tax.aom & 0 & 0 & 0 & 0 & + & 0.0316 & - & - \\
\hline & & $\begin{array}{l}\text { Taxiphyllum } \\
\text { taxirameum (Mitt.) } \\
\text { M. Fleisch. }\end{array}$ & Tax.tax & + & + & 0 & 0 & 0 & 0.777 & - & - \\
\hline 12 & $\begin{array}{l}\text { Jungermanniaceae } \\
\text { Rchb. }\end{array}$ & $\begin{array}{l}\text { Solenostoma } \\
\text { Parvitextum } \\
\text { (Amakawa) Váňa \& } \\
\text { D.G.Long* }\end{array}$ & Sol.Par & 0 & 0 & 0 & 0 & + & 0.015 & - & - \\
\hline 13 & $\begin{array}{l}\text { Lepidoziaceae } \\
\text { Limpr. }\end{array}$ & $\begin{array}{l}\text { Bazzania albifolia } \\
\text { Horik.* }\end{array}$ & Baz.alb & 0 & + & 0 & 0 & 0 & 0.3655 & - & - \\
\hline \multirow[t]{2}{*}{14} & $\begin{array}{l}\text { Leskeaceae } \\
\text { Schimp. }\end{array}$ & $\begin{array}{l}\text { Claopodium } \\
\text { rugulosifolium } \\
\text { S.Y.Zeng. }\end{array}$ & Cla.rug & 0 & + & + & 0 & 0 & 0.56 & - & - \\
\hline & & $\begin{array}{l}\text { Lindbergia } \\
\text { brachyptera (Mitt.) } \\
\text { Kindb. }\end{array}$ & Lin.bra & 0 & 0 & + & 0 & 0 & 0.1014 & - & - \\
\hline \multirow[t]{3}{*}{15} & $\begin{array}{l}\text { Leucobryaceae } \\
\text { Schimp. }\end{array}$ & $\begin{array}{l}\text { Campylopus } \\
\text { coreensis Card. }\end{array}$ & Cam.cor & + & 0 & 0 & 0 & 0 & 1.5923 & 0.800 & 0.003 \\
\hline & & $\begin{array}{l}\text { Campylopodiella } \\
\text { himalayana (Broth.) } \\
\text { J.P. Frahm }\end{array}$ & Cam.him & 0 & + & 0 & 0 & 0 & 0.325 & - & - \\
\hline & & $\begin{array}{l}\text { Leucobryum } \\
\text { bowringii Mitt. }\end{array}$ & Leu.bow & 0 & + & 0 & 0 & 0 & 1.136 & - & - \\
\hline \multirow[t]{2}{*}{16} & $\begin{array}{l}\text { Lophocoleaceae } \\
\text { Vanden Berghen }\end{array}$ & $\begin{array}{l}\text { Heteroscyphus } \\
\text { planus (Mitt.) } \\
\text { Schiffn.* }\end{array}$ & Het.pla & + & 0 & 0 & 0 & + & 0.14 & - & - \\
\hline & & $\begin{array}{l}\text { Chiloscyphus } \\
\text { horikawanus (S. } \\
\text { Hatt.) J. J. Engel \& } \\
\text { R. M. Schust.* }\end{array}$ & Chi.hor & + & 0 & 0 & 0 & 0 & 0.033 & - & - \\
\hline 17 & $\begin{array}{l}\text { Meteoriaceae } \\
\text { Kindb. }\end{array}$ & $\begin{array}{l}\text { Meteorium } \\
\text { polytrichum Dozy \& } \\
\text { Molk. }\end{array}$ & Met.pol & 0 & 0 & 0 & 0 & + & 0.2976 & - & - \\
\hline 18 & $\begin{array}{l}\text { Mniaceae } \\
\text { Schwägr. }\end{array}$ & $\begin{array}{l}\text { Pohlia } \\
\text { hyaloperistoma D. C. } \\
\text { Zhang, X. J. Li \& } \\
\text { Higuchi. }\end{array}$ & Poh.hya & 0 & + & 0 & 0 & 0 & 0.15 & - & - \\
\hline 19 & $\begin{array}{l}\text { Monosolenium } \\
\text { E.H.Wilson }\end{array}$ & $\begin{array}{l}\text { Monosolenium } \\
\text { tenerum Griff.* }\end{array}$ & Mon.ten & + & 0 & 0 & 0 & 0 & 0.801 & - & - \\
\hline 20 & $\begin{array}{l}\text { Polytrichaceae } \\
\text { Schwägr }\end{array}$ & $\begin{array}{l}\text { Atrichum crispulum } \\
\text { Schimp. ex Besch. }\end{array}$ & Atr.cri & + & 0 & 0 & 0 & 0 & 0.039 & - & - \\
\hline
\end{tabular}

Note: "*” represent liverworts 


\begin{tabular}{|c|c|c|c|c|c|c|c|c|c|c|c|}
\hline \multirow[t]{3}{*}{ No. } & \multirow[t]{3}{*}{ Family name } & \multirow[t]{2}{*}{ Species name } & \multirow[t]{2}{*}{ Abbreviation } & \multicolumn{5}{|c|}{ Location } & \multirow[t]{2}{*}{$\mathrm{TC}$} & \multirow[t]{2}{*}{ IV } & \multirow[t]{2}{*}{$p$} \\
\hline & & & & a & b & c & d & e & & & \\
\hline & & $\begin{array}{l}\text { Pogonatum neesii } \\
\text { (Müll. Hal) Dozy. }\end{array}$ & Pog.nee & + & + & 0 & 0 & 0 & 7.0256 & 0.847 & 0.002 \\
\hline \multirow[t]{16}{*}{21} & \multirow[t]{16}{*}{$\begin{array}{l}\text { Pottiaceae } \\
\text { Schimp. }\end{array}$} & $\begin{array}{l}\text { Anoectangium } \\
\text { euchloron } \\
\text { (Schwaegr.) Mitt }\end{array}$ & Ano.euc & 0 & 0 & + & + & 0 & 0.3914 & - & - \\
\hline & & $\begin{array}{l}\text { Anoectangium } \\
\text { fauriei Card }\end{array}$ & Ano.fau & 0 & 0 & + & 0 & 0 & 0.5309 & - & - \\
\hline & & $\begin{array}{l}\text { Anoectangium } \\
\text { thomsonii Mitt. }\end{array}$ & Ano.tho & + & + & 0 & 0 & 0 & 1.462 & - & - \\
\hline & & $\begin{array}{l}\text { Barbula chenia Redf. } \\
\& \text { B. C. Tan. }\end{array}$ & Bar.che & 0 & 0 & 0 & + & 0 & 0.6024 & - & - \\
\hline & & $\begin{array}{l}\text { Barbula ehrenbergii } \\
\text { (Lorentz) M.Fleisch. }\end{array}$ & Bar.ehr & 0 & + & + & + & 0 & 3.9822 & 0.509 & 0.048 \\
\hline & & $\begin{array}{l}\text { Barbula } \\
\text { propagulifera (X. J. } \\
\text { Li \& M. X. Zhang) } \\
\text { Redf. \& B. C. Tan }\end{array}$ & Bar.pro & 0 & 0 & + & 0 & 0 & 1.4938 & - & - \\
\hline & & $\begin{array}{l}\text { Bryoerythrophyllum } \\
\text { brachystegium } \\
\text { (Besch.) Saito }\end{array}$ & Bry.bra & + & 0 & + & 0 & 0 & 0.1925 & - & - \\
\hline & & $\begin{array}{l}\text { Bryoerythrophyllum } \\
\text { inaequalifolium }\end{array}$ & Bry.ina & 0 & 0 & 0 & 0 & + & 0.0825 & - & - \\
\hline & & $\begin{array}{l}\text { Chenia leptophylla } \\
\text { (Müll. Hal.) R. H. } \\
\text { Zander. }\end{array}$ & Che.lep & 0 & + & 0 & 0 & 0 & 0.306 & - & - \\
\hline & & $\begin{array}{l}\text { Didymodon } \\
\text { subandreaeoides } \\
\text { (Kindb.) R.H.Zander. }\end{array}$ & Did.sub & 0 & 0 & 0 & + & 0 & 0.084 & - & - \\
\hline & & $\begin{array}{l}\text { Didymodon tectorus } \\
\text { (Müll. Hal.) Saito }\end{array}$ & Did.tec & 0 & 0 & + & + & 0 & 1.4756 & - & - \\
\hline & & $\begin{array}{l}\text { Dymodon } \\
\text { constrictus (Mitt.) } \\
\text { Saito. }\end{array}$ & Dym.con & + & 0 & + & + & + & 4.5049 & - & - \\
\hline & & $\begin{array}{l}\text { Gymnostomum } \\
\text { aeruginosum Smith. }\end{array}$ & Gym.aer & + & 0 & 0 & 0 & 0 & 0.016 & - & - \\
\hline & & $\begin{array}{l}\text { Hydrogonium } \\
\text { consanguineum } \\
\text { (Thwait.et Mitt) Hilp }\end{array}$ & Hyd.con & + & 0 & 0 & + & 0 & 0.188 & - & - \\
\hline & & $\begin{array}{l}\text { Hydrogonium } \\
\text { laevifolium (Broth. et } \\
\text { Yas) Chen }\end{array}$ & Hyd.lae & 0 & + & 0 & 0 & 0 & 0.29 & - & - \\
\hline & & $\begin{array}{l}\text { Hydrogonium } \\
\text { majusculum (C. } \\
\text { Muell.)Chen }\end{array}$ & Hyd.maj & 0 & 0 & + & 0 & 0 & 1.428 & - & - \\
\hline
\end{tabular}

Note: “*” represent liverworts 


\begin{tabular}{|c|c|c|c|c|c|c|c|c|c|c|c|}
\hline \multirow[t]{2}{*}{ No. } & \multirow[t]{2}{*}{ Family name } & \multirow[t]{2}{*}{ Species name } & \multirow[t]{2}{*}{ Abbreviation } & \multicolumn{5}{|c|}{ Location } & \multirow[t]{2}{*}{ TC } & \multirow[t]{2}{*}{ IV } & \multirow[t]{2}{*}{$p$} \\
\hline & & & & a & b & c & d & e & & & \\
\hline & & $\begin{array}{l}\text { Hyophila spathulata } \\
\text { (Harv.) A. Jaeger }\end{array}$ & Hyo.spa & + & 0 & 0 & 0 & 0 & 0.03 & - & - \\
\hline & & $\begin{array}{l}\text { Pleuroweisia } \\
\text { schliephackei Limpr. }\end{array}$ & Ple.sch & 0 & 0 & 0 & 0 & + & 0.316 & - & - \\
\hline & & $\begin{array}{l}\text { Pseudosymblepharis } \\
\text { angustata (Mitt.) } \\
\text { Hilp. }\end{array}$ & Pse.ang & 0 & 0 & 0 & + & + & 3.0538 & 0.768 & 0.004 \\
\hline & & $\begin{array}{l}\text { Ptychomitrium } \\
\text { sinense (Mitt.) A. } \\
\text { Jaeger }\end{array}$ & Pty.sin & + & 0 & 0 & 0 & 0 & 0.184 & - & - \\
\hline & & $\begin{array}{l}\text { Tortella humilis } \\
\text { (Hedw.) Jenn. }\end{array}$ & Tor.hum & + & + & + & + & + & 4.8824 & - & - \\
\hline & & $\begin{array}{l}\text { Tortula subulata } \\
\text { Hedw. }\end{array}$ & Tor.sub & 0 & 0 & 0 & + & 0 & 0.9838 & - & - \\
\hline & & $\begin{array}{l}\text { Trichostomum } \\
\text { brachydontium } \\
\text { Bruch. }\end{array}$ & Tri.bra & + & + & 0 & + & + & 4.1366 & 0.701 & 0.005 \\
\hline & & $\begin{array}{l}\text { Trichostomum } \\
\text { crispulum Bruch. }\end{array}$ & Tri.cri & 0 & 0 & 0 & 0 & + & 0.8922 & - & - \\
\hline & & $\begin{array}{l}\text { Trichostomum } \\
\text { hattorianum B. C. } \\
\text { Tan \& Z. Iwats. }\end{array}$ & Tri.hat & 0 & + & 0 & 0 & 0 & 0.0792 & - & - \\
\hline & & $\begin{array}{l}\text { Trichostomum } \\
\text { planifolium (Dixon) } \\
\text { R. H. Zander }\end{array}$ & Tri.pla & 0 & + & 0 & + & 0 & 0.8751 & - & - \\
\hline & & $\begin{array}{l}\text { Trichostomum } \\
\text { tenuirostre (Hook. f. } \\
\text { \& Taylor) Lindb. }\end{array}$ & Tri.ten & 0 & 0 & 0 & + & 0 & 0.315 & - & - \\
\hline & & $\begin{array}{l}\text { Weissia brachycarpa } \\
\text { (Nees \& Hornsch.) } \\
\text { Jur. }\end{array}$ & Wei.bra & + & 0 & 0 & + & + & 0.925 & - & - \\
\hline & & $\begin{array}{l}\text { Weissia controversa } \\
\text { Hedw. }\end{array}$ & Wei.con & 0 & 0 & 0 & 0 & + & 2.5123 & - & - \\
\hline & & $\begin{array}{l}\text { Weissia exserta } \\
\text { (Broth.) P. C. Chen }\end{array}$ & Wei.exs & 0 & + & 0 & 0 & 0 & 0.198 & - & - \\
\hline & & $\begin{array}{l}\text { Weissia planifolia } \\
\text { Dix. }\end{array}$ & Wei.plan & 0 & 0 & 0 & + & + & 1.1465 & - & - \\
\hline & & $\begin{array}{l}\text { Weisis } \\
\text { platyphylloides Card. }\end{array}$ & Wei.plat & 0 & + & 0 & 0 & 0 & 0.1386 & - & - \\
\hline 22 & $\begin{array}{l}\text { Ptychomitriaceae } \\
\text { Schimp. }\end{array}$ & $\begin{array}{l}\text { Ptychomitrium } \\
\text { gardneri Lesq. }\end{array}$ & Pty.gar & 0 & 0 & 0 & 0 & + & 0.077 & - & - \\
\hline 23 & $\begin{array}{l}\text { Racopilaceae } \\
\text { Kindb. }\end{array}$ & $\begin{array}{l}\text { Racopilum } \\
\text { orthocarpum Wilson } \\
\text { ex Mitt. }\end{array}$ & Rac.ort & + & 0 & 0 & 0 & 0 & 0.584 & - & - \\
\hline
\end{tabular}

Note: “*” represent liverworts 


\begin{tabular}{|c|c|c|c|c|c|c|c|c|c|c|c|}
\hline \multirow[t]{2}{*}{ No. } & \multirow[t]{2}{*}{ Family name } & \multirow[t]{2}{*}{ Species name } & \multirow[t]{2}{*}{ Abbreviation } & \multicolumn{5}{|c|}{ Location } & \multirow[t]{2}{*}{$\mathrm{TC}$} & \multirow[t]{2}{*}{ IV } & \multirow[t]{2}{*}{$p$} \\
\hline & & & & a & b & c & d & e & & & \\
\hline 24 & $\begin{array}{l}\text { Sematophyllaceae } \\
\text { Broth. }\end{array}$ & $\begin{array}{l}\text { Brotherella fauriei } \\
\text { (Card.) Broth. }\end{array}$ & Bro.fau & 0 & + & 0 & 0 & 0 & 0.0693 & - & - \\
\hline \multirow[t]{4}{*}{25} & \multirow[t]{4}{*}{$\begin{array}{l}\text { Thuidiaceae } \\
\text { Schimp }\end{array}$} & $\begin{array}{l}\text { Cyrto-hypnum } \\
\text { gratum } \\
\text { (P.Beauv)Buck et } \\
\text { Crum. }\end{array}$ & Cyr.gra & 0 & 0 & 0 & 0 & + & 0.036 & - & - \\
\hline & & $\begin{array}{l}\text { Haplocladium } \\
\text { discolor (Par.et } \\
\text { Broth.) Broth. }\end{array}$ & Hap.dis & 0 & 0 & 0 & 0 & + & 0.114 & - & - \\
\hline & & $\begin{array}{l}\text { Thuidium kanedae } \\
\text { Sakurai. }\end{array}$ & Thu.kan & + & 0 & 0 & 0 & + & 0.9839 & - & - \\
\hline & & $\begin{array}{l}\text { Thuidium } \\
\text { pristocalyx (Müll. } \\
\text { Hal.) A. Jaeger. }\end{array}$ & Thu.pri & + & 0 & 0 & 0 & 0 & 0.392 & - & - \\
\hline
\end{tabular}

\subsubsection{Correlation analysis between the bryophyte community index and the contents of heavy metals in substrate}

The pearson correlation of bryophyte community Richness, Abundance index, Simpson diversity index, Shannon Wiener diversity index and Pielou evenness index with heavy metal content are shown in Fig. 5. The contents of $\mathrm{Hg}, \mathrm{Cu}, \mathrm{Zn}, \mathrm{Mn}, \mathrm{Fe}$ in the substrate were negatively correlated with the bryophyte communities index. The Shannon Wiener diversity index was positively correlated with $\mathrm{Co}$ and $\mathrm{Cr}$ but negatively correlated with other elements. With the exception of Cd, the Abundance index was negatively correlated with all other elements and the Richness was negatively correlated with all other elements besides $\mathrm{Co}$. The Simpson diversity index was positively correlated with $\mathrm{Ni}$ and $\mathrm{Cd}$, but negatively correlated with other elements, while the Pielou evenness index was positively correlated with $\mathrm{Cd}$, $\mathrm{Ni}$ and $\mathrm{Pb}$, but negatively correlated with other elements. Heavy metals can be classified into three groups according to the diversity index of the bryophyte community. The first group was $\mathrm{Fe}, \mathrm{Mn}, \mathrm{Pb}, \mathrm{Hg}, \mathrm{Ni}, \mathrm{Zn}, \mathrm{Cu}$, the second group was $\mathrm{Co}$ and $\mathrm{Cr}$ and the third group was $\mathrm{Cd}$.

\section{Discussion}

\subsection{Differences between characteristics of bryophyte communities in different locations}

Different characteristics of plant communities can reflect the environmental conditions of different regions (Pan et al., 2011; Dahwa et al., 2013; Liu Y et al., 2015), and environmental variations can also affect the distribution of plant communities (Qian et al., 2003; Pakeman et al., 2016; Fu et al., 2018; Liu et al., 2019). In mining areas, environmental damage resulting from open mining generates a reduction in bryophyte species diversity and leads to bryophyte communities dominated by short turf species that are better adapted to extreme and harsh environments (Zuo et al., 2013). In this study, the characteristics of the bryophyte communities in the five locations were quite different. In comparison to other mining areas, the number of bryophyte taxa associated with the manganese mining operation was quite large, while the diversity index was low. The higher species number may be due to limited disturbance of the bryophyte communities as the mining is carried out underground, although there are extensive damage and contamination of the environment surrounding the underground mining operation. The lower diversity index may be the result of lower 
evenness. The least number of bryophyte species and the lowest alpha diversity index were the result of human disturbance to the bryophyte community at the electrolytic plant. The electrolytic waste dump is located in the suburbs of Liaohu Town and here the alpha diversity index was higher than that of the electrolytic plant and of the mine, but lower than in the vicinity of the mine or herb-shrubland. The lower levels at these latter two sites are probably accounted for by the fact that there is only a medium level of disturbance that allows for the maintenance of relatively high diversity. The bryophyte communities at the electrolytic plant and at the electrolytic waste dump were dominated by species of Pottiaceae and Bryaceae. These species are widely distributed and most are short turfs, giving them strong adaptability to harsh environments (Zhang et al., 2012; Pang et al., 2018), consistent with bryophyte species found in other mining areas (Pan et al., 2011; Zuo et al.,2013; Wang et al., 2015). The leaves of most Pottiaceae are papillose, that is, roughened with minute protuberances, that enhance moisture uptake and storage and reflect solar radiation (Wagner et al., 2014), which may explain the dominance of Pottiaceae in the bryophyte communities at the two locations. The species composition in the vicinity of the mine and at the mine site were similar at family level, but the richness index and diversity index of bryophytes at the mine were significantly lower than in the vicinity of the mine, probably due to human activities such as mining and ore transportation which reduce species diversity.

\subsection{Differences of heavy metal contents in moss growth matrix}

There were some differences in the mean content of heavy metals. Contents of $\mathrm{Mn}, \mathrm{Fe}, \mathrm{Cr}, \mathrm{Co}, \mathrm{Ni}, \mathrm{Cu}, \mathrm{Zn}, \mathrm{Cd}, \mathrm{Hg}, \mathrm{Pb}$ in the electrolytic plant substrate were $9.79,16.53,1.31,1.97,2.06,2.39,2.59,1.062,8.55$ and 4.15 times higher respectively than the background values of those same soil elements in Guizhou Province. The high content of Mn, Cr, Cd and Fe probably derives from the dust and wastewater produced in the electrolytic process (Xu et al., 2011; Wang, 2012), while the excessive content of $\mathrm{Cu}, \mathrm{Zn}, \mathrm{Ni}$ and other elements mainly derives from road vehicle wear and traffic emissions. The contents of $\mathrm{Mn}, \mathrm{Fe}$ and $\mathrm{Cr}$ in the manganese mine were 7.02, 13.38 and 1.13 times higher than the background values of those same soil elements in Guizhou Province; this may be due to leaching of slag and rock waste from both the mining operation and from transportation. Manganese carbonate ore contains substantial quantities of $\mathrm{MnO}_{\mathrm{O}}$ and $\mathrm{Fe}_{2} \mathrm{O}_{3}$, and a small amount of $\mathrm{Cr}_{2} \mathrm{O}_{3}$ (He et al., 2013; Pan et al., 2016). Correlation analysis also shows that $\mathrm{Mn}, \mathrm{Fe}, \mathrm{Zn}, \mathrm{Cr}, \mathrm{Cu}, \mathrm{Ni}, \mathrm{Co}, \mathrm{Pb}$ and other elements have a strong positive correlation, indicating a linked relationship. Of all the elements, Fe and $\mathrm{Zn}$ contents were relatively high at all five locations, the only difference was found at the electrolytic plant, probably because of the higher contents of Fe and $\mathrm{Zn}$ in the natural geological background of this area.

\subsection{Effects of heavy metals in substrate on bryophyte communities}

Plant growth is closely related to soil composition. Excessive content of heavy metal elements in the soil affects the composition of plant communities by modifying plant growth. At all five locations, contents of $\mathrm{Hg}, \mathrm{Mn}, \mathrm{Pb}, \mathrm{Cd}$ in the substrate were the main factors affecting the distribution of bryophyte communities. This is because their content is high in most areas. Mn content in the vicinity of the mine, at the mine site, at the electrolytic plant and at the electrolytic waste dump were much higher than background values for soil in Guizhou Province; $\mathrm{Hg}$ content at the mine, at the electrolytic plant and at the electrolytic waste dump were much higher than Guizhou background value. Pb and Cd contents were much higher than Guizhou background values in the vicinity of the mine but were similar to Guizhou background values at the other four locations. As the concentration of $\mathrm{Hg}$ increased in the substrate, there was a corresponding increase in the number of bryophyte species with strong tolerance to $\mathrm{Hg}$, but there was a decline in bryophyte species richness and diversity (Liu et al., 2011; Liu et al., 2018). Hg has a negative effect on the distribution of bryophyte communities (Fig. 5), consistent with previous research results (Liu et al., 2011; Liu et al., 2018). Mn is toxic to plants and this is one of the most concerning environmental problems generated by manganese mining and processing. Excessive content of $\mathrm{Mn}$ can interfere with plant metabolism and restrict plant growth and development (Boojar et al., 2008). Relatively few specially adapted plants have a tolerance to high levels of manganese (Boojar et al., 2008). Mn content in the substrate underlying bryophyte communities at both the manganese mine and electrolytic plant were found to be very high, resulting in a significant impact on bryophyte community distribution. Mn tolerant bryophyte species were mainly distributed around the

Page $15 / 25$ 
electrolytic plant and Manganese mine, while those not Mn tolerant mainly occurred in the vicinity of the mine and in herbshrubland. $\mathrm{Pb}$ and $\mathrm{Cd}$ appear not to have impacted the growth of bryophytes (Fig. 4), probably because of the low content of these metals. Most bryophyte species have poor tolerance to heavy metals, but some species such as Didymodon tectorus, Barbula ehrenbergii and Barbula propagulifera show strong tolerance and were well able to tolerate $\mathrm{Mn}$ and $\mathrm{Pb}$; Hydrogonium majusculum, Didymodon constrictus, and Anomobryum julaceum were able to tolerate Pb and Cd; Bryum pallescens and Ditrichum pallidum were well able tolerate to $\mathrm{Hg}$ (Fig. 4). Bryophytes can be effective indicators of heavy metals in the environment: gold deposits have been discovered as a result of gold enrichment of aquatic bryophytes; and copper deposits have been located by the presence of Mielichhoferia elongata which is referred to as a copper moss because of its usual association with copper-rich environments. Barbula propagulifera showed the strongest tolerance to $\mathrm{Pb}$ and $\mathrm{Mn}$ amongst all the indicator species at each of the five locations, identifying the species as the most effective moss to utilize as an indicator of $\mathrm{Mn}$ and $\mathrm{Pb}$ pollution.

\section{Conclusion}

There were significant differences in the characteristics of bryophyte communities at each of the five locations. The richness index was in the order: vicinity of the mine $>$ herb-shrubland > mine site > electrolytic waste dump > electrolytic plant; whereas the alpha diversity index was in the order: herb-shrubland > vicinity of the mine > electrolytic waste dump > mine site > electrolytic plant. The species composition of each location was quite different at the family level. The vicinity of the mine and the mine site were dominated by Hypnaceae and Polytrichaceae respectively, whereas Pottiaceae species dominated the electrolytic plant, electrolytic waste dump and the herb-shrubland. These differences are related to human activities, principally mining and ore processing, both of which will generate substantial quantities of heavy metal elements.

CCA analysis showed that the distribution of bryophyte communities was principally determined by the levels of $\mathrm{Hg}, \mathrm{Mn}$, $\mathrm{Pb}$ and $\mathrm{Cd}$ in the underlying substrates. $\mathrm{Hg}$ and $\mathrm{Mn}$ restrict the distribution and reduce the diversity of bryophyte communities. Bryophyte communities were not limited by levels of $\mathrm{Pb}$ and $\mathrm{Cd}$, since the levels of those metals were relatively low and did not exceed the published background values for Guizhou. Barbula propagulifera was found to have the greatest tolerance to $\mathrm{Mn}$ and $\mathrm{Pb}$ concentration in the substrate and is practical to use as an indicator species of $\mathrm{Mn}$ and $\mathrm{Pb}$ pollution.

\section{Declarations}

- Ethics approval and consent to participate

Not applicable

- Consent for publication

Not applicable

- Availability of data and materials

All data generated or analysed during this study are included in this published article [and its supplementary information files].

- Competing interests

The authors declare that they have no known competing financial interests or personal relationships that could have appeared to influence the work reported in this paper.

Page 16/25 
- Funding

This work was supported by the National Nature Science Foundation of China (No.31960044), the Department of Science and Technology Foundation of Guizhou Province, China [DSTFGC, (2019)] and the Science and Technology Cooperation Program of Guizhou Procince (Qiankehe LH[2015]7778).

- Authors' contributions

He Chunmei: Conceptualization, Methodology, Software, Formal analysis, Data curation, Writing-original draft, Writingreview \& editing, Visualization. Zhang Zhaohui: Methodology, Validation, Resources, Writing-review \& editing, Supervision, Project administration, Funding acquisition. Wang Zhihui: Writing-review \& editing, Supervision. Shi Kuangzheng:

Software, Writing-review \& editing. Wu Qimei: Project administration, Funding acquisition. Wang Dengfu: Software, Writingreview \& editing.

- Acknowledgements

We would like to thank Alison Downing from the Department of Biological Sciences, Macquarie University, Sydney, for constructive comments and advice on the text. And thanks to Wang Tao for help with field work.

\section{References}

Bargagli, R., 2016. Moss and lichen biomonitoring of atmospheric mercury: A review. Science of The Total Environment 572: 216-231. http://doi.10.1016/j.scitotenv.2016.07.202.

Boojar, M.M.A., Goodarzi, F., 2008. Comparative evaluation of oxidative stress status and manganese availability in plants growing on manganese mine. Ecotoxicology \& Environmental Safety 71:692-699. http://doi.10.1016/j.ecoenv.2007.10.011

Büscher, P., Koedam, N., Speybrock, D.V., 1990. Cation-exchange properties and adaptation to soil acidity in bryophytes. New Phytologist 115: 177-186. http://doi.10.1111/j.1469-8137.1990.tb00936.x.

Chen, S.F., Wu, D.F., Wu, J.N., 1989. Using lichen communities as SO2 pollution monitors. Journal of Nanjing Normal University (Natural Science) 12: 77-82. http://doi.cnki:sun:njsf.0.1989-04-013 (In Chinese).

Chinese Mineral Deposits Editorial Committee (1989). Chinese Mineral Deposits. Vol. 1. Geological Publishing House, Beijing.

Cowden, P., Aherne, J., 2019. Assessment of atmospheric metal deposition by moss biomonitoring in a region under the influence of a long standing active aluminium smelter, Atmospheric Environment 201: 84-91.

http://doi.10.1016/j.atmosenv.2018.12.022.

Dahwa, E., Mudzengi. C.P., Hungwe, T., 2013. Influence of grazing intensity on soil properties and shaping herbaceous plant communities in semi-arid Dambo Wetlands of Zimbabwe. Journal of Environmental Protection 4:1181-1188. http://doi.10.4236/jep.2013.410135.

Denaye, F.O., Haluwyn, C.V., Bruno de Foucault, Schumacker, R., Colein, P., 1999. Use of bryological communities as a diagnostic tool of heavy metal soil contamination (Cd, Pb, Zn) in Northern France. Plant Ecology 140: 191-201. http://doi.10.1023/A:1009771209869.

Deng, W.B., Zhang, Y.W., Kong, L.H., Shang, L., 2019. Current status of manganese ore resources in China and selecting for national physical geological data of manganese ore deposits. China Mining Magazine 28: 175-182.

http://doi.10.12075/j.issn.1004-4051.2019.09.001 (In Chinese)

Page $17 / 25$ 
Fu, Z.Y., Jiang, H., Wang, G.Q., A, Y.L., Xue, B.L, Wang, H.Q., 2018. Effects of soil properties on

plant community structure in a semi-arid grassland. Chinese Journal of Ecology 37: 823-830.

https://doi.org/10.13292/j.1000-4890.201803.040 (In Chinese).

Gao, J.B., Yang, R.D., Xu, H., Zhang, X., Feng, K.N., Zheng, L.L., 2018. Genesis of Permian sedimentary manganese deposits in Zunyi, Guizhoug Province, SW China: Constraints from geology and elemental geochemistry. Journal of Geochemical Exploration 192: 142-154. https://doi.org/10.1016/j.gexplo.2018.06.012

Gao, Q., 1994. Flora Bryophytarum Sinicorum Vol. 1. Science Press, Beijing.

Gao, Q., 1996. Flora Bryophytarum Sinicorum Vol. 2. Science Press, Beijing.

Gao, Q., Wu, Y.H., 2010. Genera Hepaticopsida et Anthocerotopsida Sinicorum. Science Press, Beijing.

Gerdol, R., Bragazza, L., Marchesini, R., Medici, A., Pedrini, P., Benedetti, S., et al., 2002. Use of moss (Tortula muralis Hedw.) for monitoring organic and inorganic air pollution in urban and rural sites in Northern Italy. Atmospheric Environment 36: 4069-4075. http://doi.10.1016/S1352-2310(02)00298-4.

Guo, Y., Wang, Z.H., Zhang, Z.H., 2017. Ability of Brachymenium exile to monitor metal elements at an electrolytic aluminium factory in karst mountainous area. Environmental Science \& Technology 40: 148-152.

http://doi.10.3969/j.issn.1003-6504.2017.03.024 (In Chinese).

He, Z.W., Yang, R.D., Gao, J.B., Cheng, W., Zhang, R.B., Zhang, P.Y., 2013. Sedimentary geochemical characteristics of manganese deposits in Xixibao, Songtao County, Guizhou Province. Geochimica 42: 576-588. http://doi.10.19700/j.03791726.2013.06.006 (In Chinese).

Hu, R.L., Wang, Y.F., 2005. Flora Bryophytarum Sinicorum Vol. 7. Science Press, Beijing.

Jia, Y., He, S., 2013. Species Catalogue of China Vol. 1. Plants: Bryophytes. Science Press, Beijing.

Li, C.Y., Zhang, Z.H., Wang, Z.H., Wu, Q.M., 2020. Effects of environmental factors and heavy metals on the vertical distribution of bryophytes in sinkhole environment. Plant Biology. http://doi.10.1111/PLB.13129

Li, X.J., 2000. Flora Bryophytarum Sinicorum Vol. 3. Science Press, Beijing.

Li, X.J., 2006. Flora Bryophytarum Sinicorum Vol. 4. Science Press, Beijing.

Liu, J.Y., Dou, S.Q., Xiao, J.Z., Zhang, J., Pan, W., 2019. Environmental impacts evaluation of mineral resources development during mining life cycle: a case study on Songtao manganese integrated exploration area in Guizhou. Resources and Industries 21: 36-43. http://doi.10.13776/j.cnki.resourcesindustries.20190605.004 (In Chinese).

Liu, R.X., Wang, Z.H., Zhang, Z.H., 2011. Ecological monitoring of bryophytes for mercury pollution in Danzhai Mercury Mine Area囚Guizhou Province『China. Ecologica Sinica 31: 1558-1566. http://doi.cnki:sun:stxb.0.2011-06-010 (In Chinese).

Liu, R., Zhang, Z.H., Shen, J.C., Wang, Z.H., 2018. Community characteristics of bryophyte in karst caves and its effect on heavy metals pollution: a case of Zhijin Cave, Guizhou Province. Biodiversity Science 26: 1277-1288. http://doi.cnki:sun:swdy.0.2018-12-003 (In Chinese).

Liu, R., Zhang, Z.H., Shen, J.C., Wang, Z.H., 2019. Bryophyte diversity in karst sinkholes affected by different degrees of human disturbance. Acta Societatis Botanicorum Poloniae 88: 3620. https://doi.org/10.5586/asbp.3620 
Liu, Y., Pi, C.Y., Tian, S., 2015. Relationships between characteristics of ground bryophyte communities and environmental factors in urban area of Chongqing, China. Chinese Journal of Applied Ecology 26: 3145-3152. http://doi.10.13287/j.10019332.20150921.001 (In Chinese)

Liu, Z.N., Xu, H., Wang, Q.S., Chen, M., 2015. China's Manganese supply-demand actuality and its sustainable development. Resources and Industries 17: 38-43. http://doi.10.13776/j.cnki.resourcesindustries.20151126.006. (In Chinese).

Muhammad, S., Shah, M.T., Khan, S., 2011. Health risk assessment of heavy metals and their source apportionment in drinking water of Kohistan region, Northern Pakistan. Microchemical Journal 98: 334-343.

http://doi.10.1016/j.microc.2011.03.003.

Pakeman, R.J., Alexander, J., Brooker, R.W., Cummins, R., Fielding, D.A., Gore, S., et al., 2016. Long-term impacts of nitrogen deposition on coastal plant communities. Environmental Pollution 212: 337-347. http://doi.10.1016/j.envpol.2016.01.084.

Pan, W., Zhuo, X.Z., Chen, X.S., Yang, S.T., Zhao, S., 2016. Geochemical characteristics of manganese bearing rock series in black shale basins of Northeast Guizhou, China. Acta Sedimentologica Sinica 34: 868-880.

http://doi.10.14027/j.cnki.cjxb.2016.05.006 (In Chinese).

Pan, S., Zhang, Z.H., 2011. Bryophyte communities from abandoned mercury mine in Eastern Guizhou Province. Plant Research 31: 241-248. http://doi.cnki:sum:mbzw.0.2011-02-020 (In Chinese).

Pang, J.P., Wang, Z.H., Zhang, Z.H., 2018. Characteristics of bryophyte communities and their successional patterns in different habitats from karst dolomite rocky desertification areas. Ecological Science 37: 59-66.

http://doi.10.14108/j.cnki.1008-8873.2018.03.008 (In Chinese).

Qian, Y.B., Jiang, J., Wu, Z.N., 2003. Soil heterogeneity and its impact on ecological distribution of plant community in the Aiby Lake area. Arid Land Geography 03: 27-32. http://doi.10.3321/j.issn:1000-6060.2003.03.004 (In Chinese).

Rao, D., 1982. Responses of Bryophytes to Air Pollution. Bryophyte Ecology 12: 445-471, http://doi.10.1007/978-94-0095891-312.

Richardson, 1981. The Biology of Mosses. Oxford: Blackwell Scientific Publications. http://doi.10.2307/3243016.

Salo, H., Buckko, M.S., Vaahtovuo, E., Limo, J., Mäkinen, J.K., Pesonen, L.J., 2012. Biomonitoring of air pollution in SW Finland by magnetic and chemical measurements of moss bags and lichens. Journal of Geochemical Exploration 115: 6981. http://doi.10.1016/j.gexplo.2-12.02.009

Samecka, C.A., Kempers, A.J., 1998. Bioindication of gold by aquatic bryophytes. Acta Hydrochimica et Hyd-robiologica 26: 90-94. http://doi.10.1002/(SICI)1521-401X(199803)26:23.0.CO;2-Q

Schintu, M., Cogoni, A., Durante, L., Cantaluppi, C., Contu, A., 2005. Moss (Bryum radiculosum) as a bioindicator of trace metal deposition around an industrialised area in Sardinia (Italy). Chemosphere 60: 610-618.

http://doi.10.1016/j.chemosphere.2005.01.050

Vincent, C.D., Lawlor, A.J., Tipping, E., 2001. Accumulation of Al, Mn, Fe, Cu, Zn, Cd and Pb by the bryophyte Scapania undulata in three upland waters of different pH. Environmental Pollution, 114: 93-100. http://doi.10.1016/S02697491(00)00201.

Vuković, G., Urošević, M.A., Razumenić, I., Goryainova, Z., Frontasyeva, M., Tomašević, M., et 
al, 2013. Active moss biomonitoring of small-scale spatial distribution of airborne major and trace elements in the Belgrade urban area. Environmental Science and Pollution Research 20: 5461-5470. http://doi.10.1007/s11356-013-15619.

Wagner S., Bader M.Y., Zotz, G., 2014. Physiological Ecology of Tropical Bryophytes. In: Hanson D., Rice S. (eds) Photosynthesis in Bryophytes and Early Land Plants, Advances in Photosynthesis and Respiration (Including Bioenergy and Related Processes), Vol. 37. Springer, Dordrecht. http://doi.10.1007/978-94-007-6988-515.

Wang, S.Q., Zhang, Z.H., Wang, Z.H., 2015. Bryophyte communities as biomonitors of environmental factors in the Goujiang karst bauxite, southwestern China. Science of The Total Environment 538: 270-278. http://doi.10.1016/j.scitotenv.2015.08.049

Wang, Y.Y., 2012. Human health risk study of selenium and heavy metals pollution in electrolysis manganese industry. Anhui University of Science and Technology. http://doi.10.7666/d.y2133716 (In Chinese).

Wu, M.K., Cao, T., Zhang, X.P., 2008. Biomonitoring Pollution of Atmospheric Heavy Metal by Moss Bag in Tongling City, Anhui Province. Acta Laser Biology Sinica 17: 554-558. http://doi.1007-7146(2008)04-0554-05 (In Chinese).

Wu, P.C., 1998. Bryophyte Biology. Science Press, Beijing.

Wu, P.C., Jia, Y., 2011. Flora Bryophytarum Sinicorum Vol. 5. Science Press, Beijing.

Wu, P.C., 2011. Flora Bryophytarum Sinicorum Vol. 6. Science Press, Beijing.

Wu, P.C., Jia, Y., 2004. Flora Bryophytarum Sinicorum Vol. 8. Science Press, Beijing.

Wu, Y.H., Huang, G.H., Gao, Q., 2001. Research advance in response and adaption of bryophytes to environmental change. Chinese Journal of Applied Ecology 12: 943-946. http://doi.10.1088/0256-307X/18/11/313 (In Chinese).

Xu, L.J., Wang, X.M., Chen, H.C., Liu, C.L., 2011. Mn forms and environmental impact of electrolytic manganese residue. Advanced Materials Research 183-185: 570-574. http://doi.10.4028/www.scientific.net/AMR.183-185.570.

Xu, M.Q., Cao, H., 2004. Ecological basis and application of PFU protozoan community in bio-monitoring with relation to water quality. Acta Ecologica Sinica 24: 1540-1547. http://doi.cnki:sun:stxb.0.2004-07-031 (In Chinese).

Yang, Z.C., Wang, Z.H., Zhang, Z.H., 2011. Biomonitoring of testate amoebae (protozoa) as toxic metals absorbed in aquatic bryophytes from the Hg-TI mineralized area (China). Environmental Monitoring \& Assessment 176: $321-329$. http://10.1007/s10661-010-1585-2.

Zhang, X.Q., Zeng, J.J., Chen, J.W., Luo, Z.W., Sun, M., 2012. The saxicolous moss's features of absorbing water and its structural adaptability in the heterogeneous environment with rock desertification. Acta Ecologica Sinica 32: $3902-3911$. http://doi.10.5846/stxb201111231792 (In Chinese)

Zuo, J.B., 1990. A numerical analysis of Flora. Acta Botanica Yunnanica 12: 179-185. http://doi.cnki:sun:yoke.0.1990-02009 (In Chinese).

Zuo, S.Y., Wang, Z.H., Zhang, Z.H., 2013. Bryophyte species diversity and its indicative roles in monitoring heavy metals pollution in Zhangjiawanzi gold deposit area of Guizhou Province, Southwest China. Chinese Journal of Ecology 32: 412417. http://doi.cnki:sun:stxz.0.2013-02-030 (In Chinese).

\section{Figures}

Page 20/25 

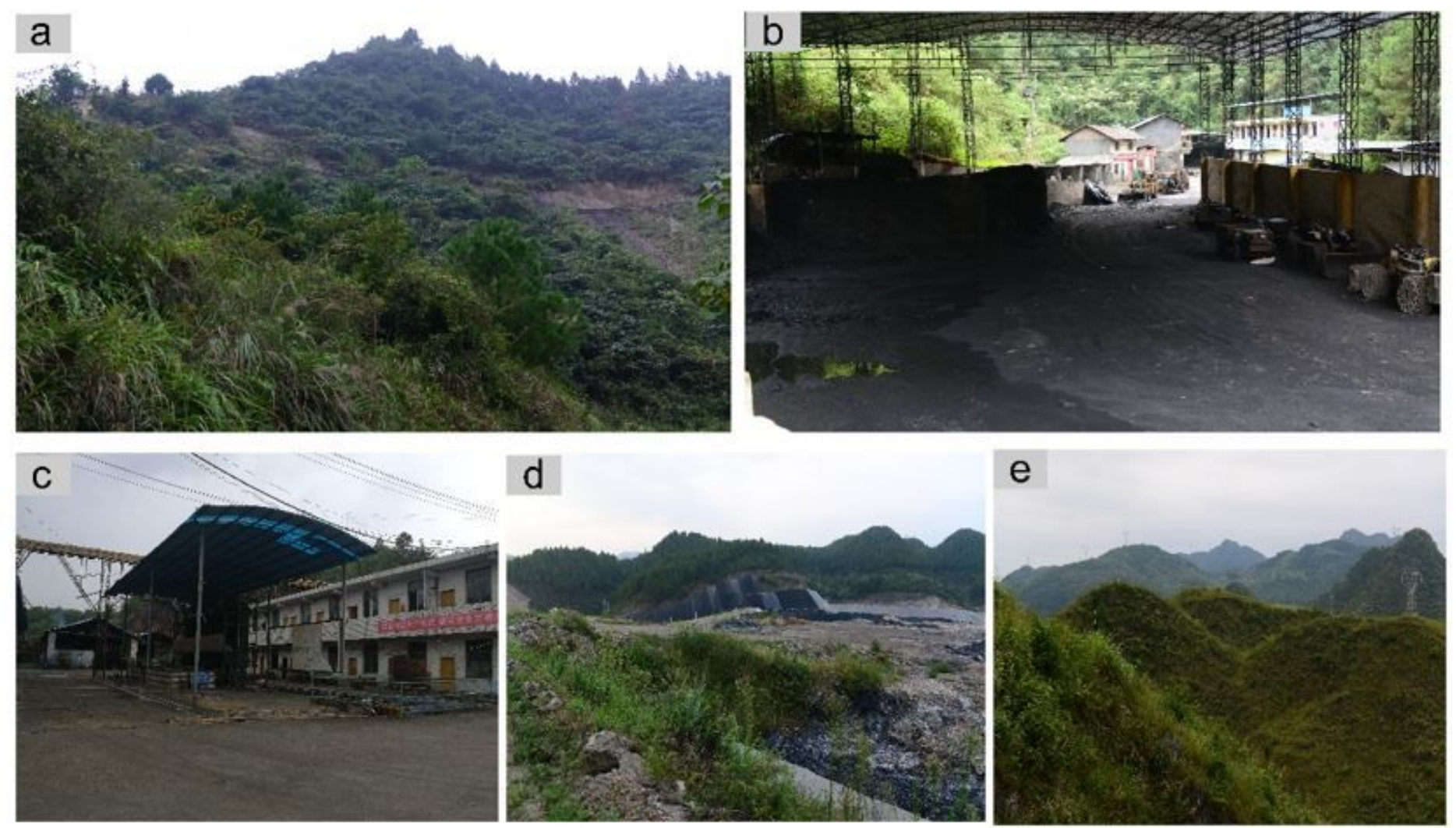

\section{Figure 1}

a: Vicinity of the mine (a1-a5); b: Mine site (b1-b5); c: Electrolytic plant (c1-c5); d: Electrolytic waste dump (d1-d5); e: Herbshrubland (e1-e5). 
(A)

(B)
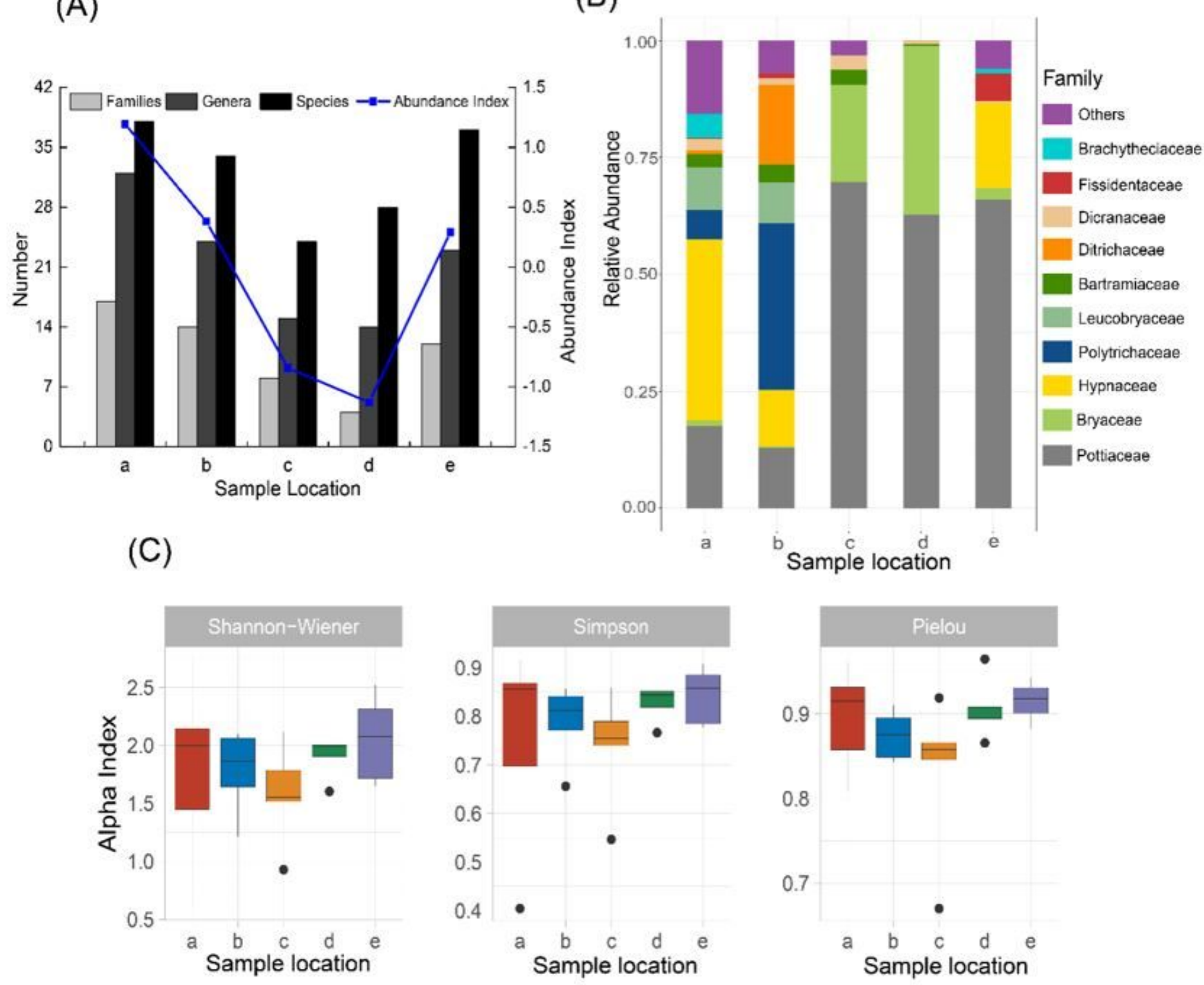

Figure 2

(A) Number of bryophyte families, genera and species together with abundance index for each of the five locations. (B) Relative abundance of bryophytes species at the family level at each location. (C) Alpha diversity of bryophyte communities at the five locations: a. Vicinity of the mine; b. Mine site; c. Electrolytic plant; d. Electrolytic waste dump; e. Herb-shrubland 


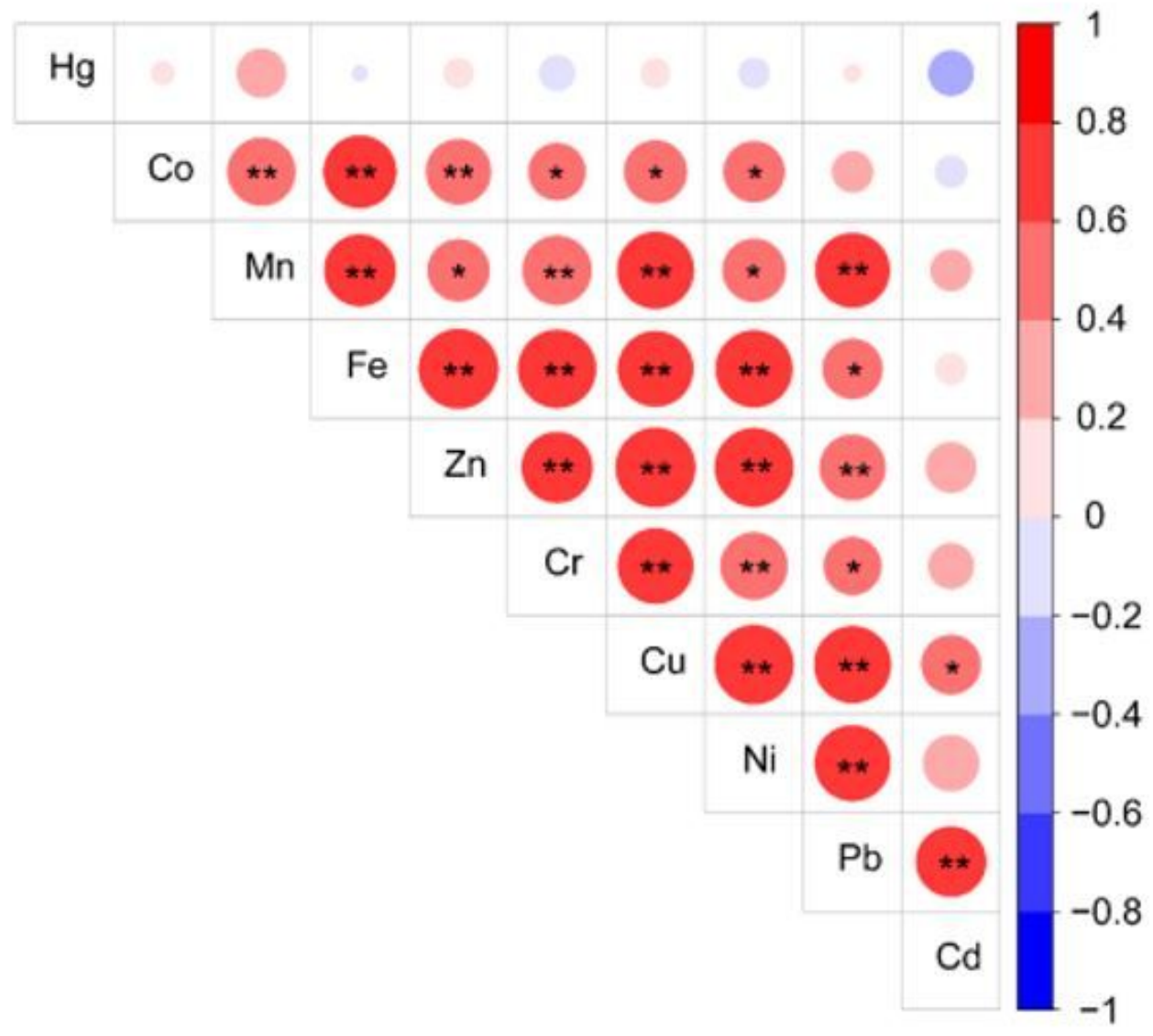

Figure 3

Correlation of heavy metals content in substrate **indicates significant correlation at 0.01 level (bilateral); * indicates significant correlation at 0.05 level (bilateral). 


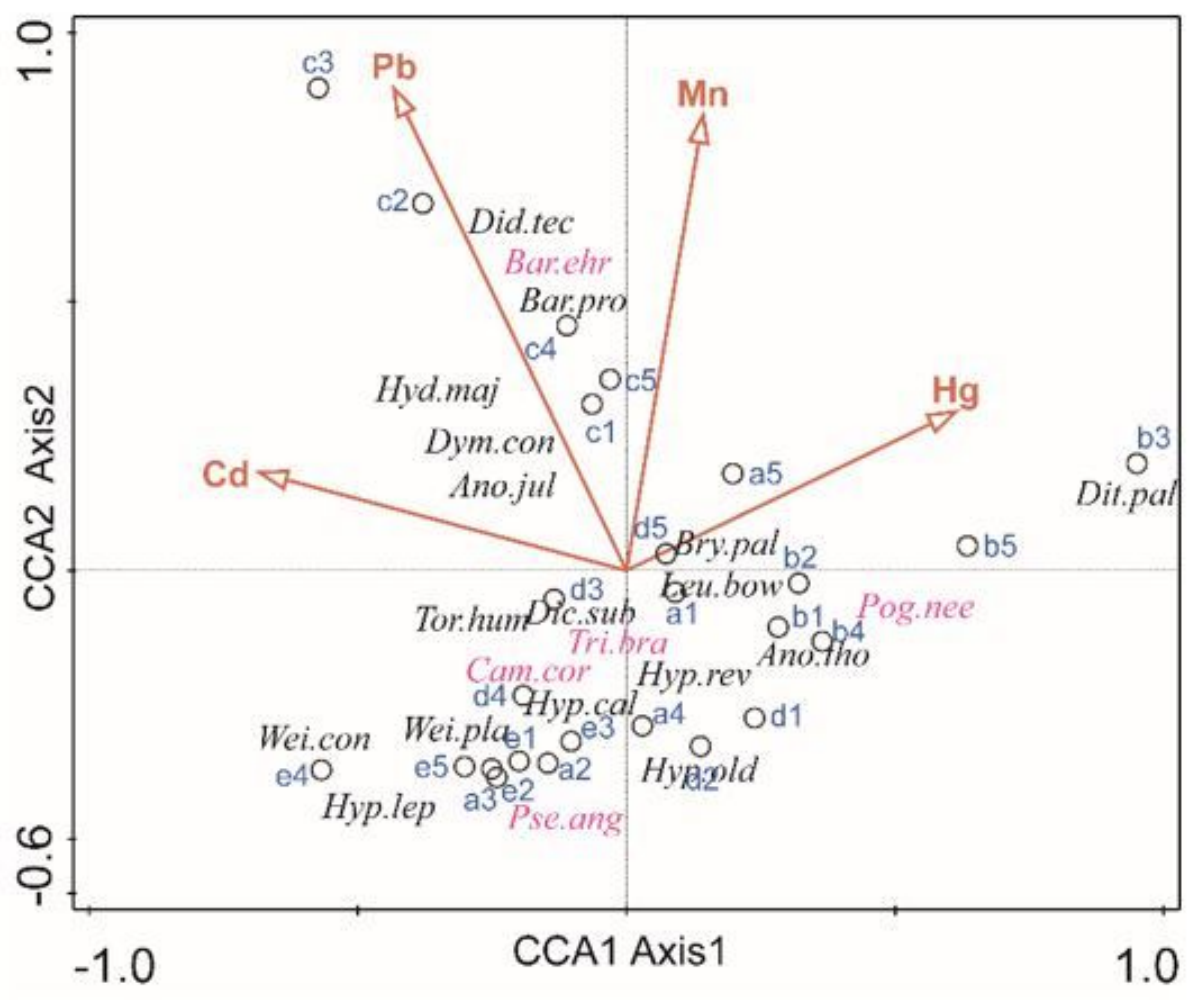

\section{Figure 4}

Relationship between heavy metal content in the substrate and distribution of bryophyte communities at the five locations. a1-a5: Vicinity of the mine; b1-b5: Mine site; c1-c5: Electrolytic plant; d1-d5: Electrolytic waste dump; e1-e5: Herb-shrubland. 


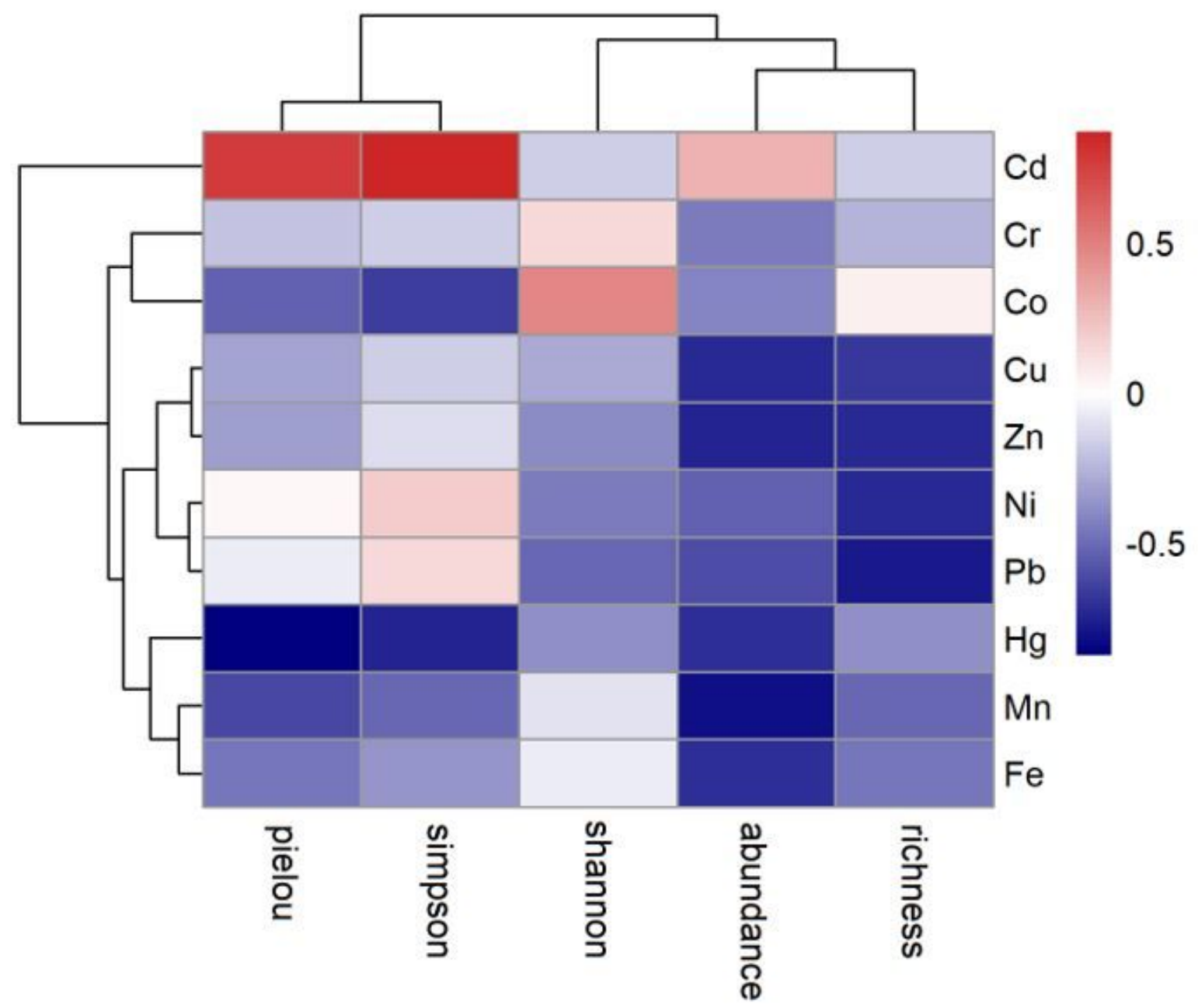

Figure 5

Heatmap of correlation between bryophyte communities and heavy metals

\section{Supplementary Files}

This is a list of supplementary files associated with this preprint. Click to download.

- formulas.docx 